B. A. Datskovsky

Nagoya Math. J.

Vol. 142 (1996), 95-132

\title{
ON DIRICHLET SERIES WHOSE COEFFICIENTS ARE CLASS NUMBERS OF BINARY QUADRATIC FORMS*
}

\author{
BORIS A. DATSKOVSKY
}

\section{Introduction}

0.1. For an integer $d>0$ (resp. $d<0$ ) let $h_{d}$ denote the number of $S l_{2}(\mathbf{Z})$ equivalence classes of primitive (resp. primitive positive-definite) integral binary quadratic forms of discriminant $d$. For $d>0$ let $\varepsilon_{d}=\frac{1}{2}(t+u \sqrt{d})$ where $t$ and $u$ are the smallest positive integral solutions of the equation $t^{2}-d u^{2}=4$ if $d$ is a non-square and $\varepsilon_{d}=1$ if $d$ is a square. For $d<0$ let $w_{d}$ denote the number of roots of unity in the quadratic field $\mathbf{Q}(\sqrt{d})$. Define the Dirichlet series

$$
\begin{aligned}
& \xi_{+}(s)=\zeta(2 s) \sum_{d=1}^{\infty} \frac{h_{d} \log \varepsilon_{d}}{d^{s}} \\
& \xi_{-}(s)=2 \zeta(2 s) \sum_{-d=1}^{\infty} \frac{h_{d} w_{d}^{-1}}{(-d)^{s}} \\
& \xi_{+}^{*}(s)=\zeta(2 s)\left\{\sum_{d=1}^{\infty} \frac{h_{4 d} \log \varepsilon_{4 d}}{(4 d)^{s}}+2^{-2 s} \sum_{d=1}^{\infty} \frac{h_{4 d+1} \log \varepsilon_{4 d+1}}{(4 d+1)^{s}}\right\} \\
& \xi_{-}^{*}(s)=2 \zeta(2 s)\left\{\sum_{-d=1}^{\infty} \frac{h_{4 d} w_{4 d}^{-1}}{(-4 d)^{s}}+2^{-2 s} \sum_{-d=1}^{\infty} \frac{h_{4 d+1} w_{4 d+1}^{-1}}{(-4 d-1)^{s}}\right\} .
\end{aligned}
$$

Shintani ([12], Theorem 2) discovered that the series $\xi_{ \pm}(s)$ and $\xi_{ \pm}^{*}(s)$ satisfy a curious functional equation

$$
\left(\begin{array}{c}
\xi_{+}\left(\frac{3}{2}-s\right) \\
\xi_{-}\left(\frac{3}{2}-s\right)
\end{array}\right)=2^{2 s-1} \pi^{\frac{1}{2}-2 s} \Gamma\left(s-\frac{1}{2}\right) \Gamma(s)\left(\begin{array}{cc}
\sin \pi s & \pi \\
0 & \cos \pi s
\end{array}\right)\left(\begin{array}{l}
\xi_{+}^{*}(s) \\
\xi_{-}^{*}(s)
\end{array}\right)
$$

Received November 8, 1994.

Revised April 6, 1995.

* Research supported by SFB-170, Göttingen, Germany and by a Fulbright fellowship 


$$
+2^{-1} \pi^{\frac{1}{2}-2 s} \Gamma\left(s-\frac{1}{2}\right) \Gamma(s) \zeta(2 s-1)\left(\begin{array}{c}
\sin \pi s T(s) \\
-1
\end{array}\right)
$$

where

$$
\begin{aligned}
T(s)= & \left(\frac{\Gamma^{\prime}}{\Gamma}(s)-\frac{\Gamma^{\prime}}{\Gamma}\left(s-\frac{1}{2}\right)\right)+2\left(\frac{\zeta^{\prime}}{\zeta}(2 s)-\frac{\zeta^{\prime}}{\zeta}(2 s-1)\right) \\
& -2\left(\frac{\zeta^{\prime}}{\zeta}(3-2 s)-\frac{\zeta^{\prime}}{\zeta}(2-2 s)\right)+\frac{\log 2}{1-2^{-2 s}} .
\end{aligned}
$$

In an excellent recent paper [8] H. Saito extended Shintani's result to a family of $L$-functions associated with the space of binary quadratic forms with coefficients in $\mathbf{Q}$. The purpose of this paper is to establish Shintani's functional equation for a family of zeta functions associated with the space of binary quadratic forms with coefficients in an algebraic number field.

0.2. Let $K$ be an algebraic number field. Denote by $M(K)$ the complete set of places of $K$. For $\nu \in M(K)$, let $K_{\nu}$ denote the completion of $K$ at $\nu$. If $\nu$ is non-archimedean, let $q_{\nu}$ denote the size of the residue field of $K_{\nu}$.

Let $L$ be a quadratic extension of $K$. We call $L \otimes K_{\nu}$ the $\nu$-splitting type of $L$. We say that two fields $L$ and $L^{\prime}$ have the same $\nu$-splitting type if $L \otimes K_{\nu} \cong L^{\prime} \otimes$ $K_{\nu}$ as a $K_{\nu}$-algebra. Clearly, the number of $\nu$-splitting types is finite. For example, if $K_{\nu}=\mathbf{R}$, then $L \otimes K_{\nu}$ is either $\mathbf{R} \oplus \mathbf{R}$ or $\mathbf{C}$ (2 splitting types), and if $K_{\nu}=\mathbf{C}$, then $L \otimes K_{\nu}=\mathbf{C} \oplus \mathbf{C}$ (1 splitting type).

If $\nu$ is non-archimedean and $q_{\nu}$ is not a power of 2 , then $L \otimes K_{\nu}$ is either $K_{\nu} \oplus K_{\nu}$ (split case), a quadratic unramified extension of $K_{\nu}$, or one of the two quadratic ramified extensions of $K_{\nu}$; thus the number of $\nu$-splitting types is 4 . If $q_{\nu}$ is a power of 2 , then the number of quadratic ramified extensions of $K_{\nu}$ is, unfortunately, larger than 2. In this case, we will only distinguish between two quadratic ramified extensions of $K_{\nu}$ if their discriminants have different absolute norms.

Let $\mathrm{X}_{\nu}$ denote the set of all $\nu$-splitting types of quadratic extensions of $K$. For a finite set $S$ of places of $K$, set $\mathrm{X}_{S}=\Pi_{\nu \in S} \mathrm{X}_{\nu}$. Let $\mathrm{x}_{S}=\left(\mathrm{x}_{\nu}\right)_{\nu \in S} \in \mathrm{X}_{S}$. We will say that $L$ has an $S$-splitting signature $\mathrm{x}_{S}$ if $L$ has splitting type $\mathrm{x}_{\nu}$ at every $\nu \in S$. In this case we will write $L \sim \mathrm{x}_{S}$.

Denote by $D_{L}$ the absolute norm of the discriminant of $L$ and by $D_{L / K}$ the norm of the relative discriminant of $L$ over $K$. Let $\zeta_{L}(s)$ denote the Dedekind zeta function of $L$ and let $\rho_{L}=D_{L}^{1 / 2} \operatorname{Res}_{s=1} \zeta_{L}(s)$.

Let $S$ be a finite set of places of $K$ containing all the infinite places. In [3] we 
encountered the Dirichlet series

$$
\xi_{\mathrm{x}_{S}}(s)=\sum_{L \sim \mathrm{x}_{S}} \frac{\rho_{L}}{D_{L / K}^{s}} \eta_{L, S}(s)
$$

where

$$
\eta_{L, S}(s)=\frac{\zeta_{K, S}(2 s-1) \zeta_{K, S}^{2}(2 s)}{\zeta_{L, S}(2 s)}
$$

Here $\zeta_{K, S}(s)$ denotes the truncated Dedekind zeta function $\zeta_{K, S}(s)=\Pi_{\nu \notin S}\left(1-q_{\nu}^{-s}\right)^{-1}$, and $\zeta_{L, S}(s)=\Pi_{\omega \in M(L), w \mid \nu \notin S}\left(1-q_{\omega}^{-s}\right)^{-1}$. If $K=\mathbf{Q}$ and $S$ consists of the infinite place of $\mathbf{Q}$, the series of $(0.2)$ differ from the series $\xi_{ \pm}(s)$ of Shintani only by a constant factor (see [3], Theorem 0.2).

The series $\xi_{\mathrm{x}_{s}}(s)$ satisfy functional equations

$$
\xi_{\mathrm{x}_{s}}\left(\frac{3}{2}-s\right)=D_{K}^{2 s-\frac{3}{2}} \sum_{\mathrm{y}_{s}} \Gamma_{\mathrm{x}_{s} \mathrm{y}_{s}}(s) \xi_{\mathrm{y}_{s}}^{*}(s)+T_{\mathrm{x}_{s}}(s)
$$

(see Theorem 1.2).

We will not define $\xi_{\mathrm{y}_{s}}^{*}(s)$ explicitly in the introduction. It suffices to say that

$$
\xi_{\mathrm{y}_{S}}^{*}(s)=\sum_{L \sim \mathrm{y}_{S}} \frac{\rho_{L}}{D_{L / K}^{s}} \eta_{L, S}^{*}(s)
$$

where $\eta_{L, S}^{*}(s)$ is given by an Euler product that differs from the Euler product of $\eta_{L, S}(s)$ only at those $\nu \notin S$ that lie over 2 . In particular, if $S$ contains all places of $K$ that lie over 2 , then $\xi_{\mathrm{y}_{s}}^{*}(s)=\xi_{\mathrm{y}_{s}}(s)$.

The object of this paper is to compute the functional equation coefficients $\Gamma_{\mathrm{x}_{s} \mathrm{y}_{s}}(s)$ and the remainder $T_{\mathrm{x}_{s}}(s)$.

0.3. This paper is based on an earlier study [3] of zeta functions associated with the space of binary quadratic forms. The fact that the series $\xi_{\mathrm{x}_{s}}$ satisfy the functional equation (0.3) easily follows from the theory of zeta functions associated with prehomogeneous vector spaces. Moreover, the coefficient matrix $\left(\Gamma_{\mathrm{x}_{S} \mathrm{y}_{s}}(s)\right)=\Pi_{\nu \in S}\left(\Gamma_{\mathrm{x}_{\nu} \mathrm{y}_{\nu}}(s)\right)$, and the local coefficient matrix $\left(\Gamma_{\mathrm{x}_{\nu} \mathrm{y}_{\nu}}(s)\right)$ is precisely the functional equation matrix for the local zeta functions associated with the prehomogeneous vector space of binary quadratic forms. The local functional equation for zeta functions associated with prehomogeneous vector spaces has lately been a subject of considerable mathematical interest. In [2] I computed the local functional equation matrix for the space of binary cubic forms with coefficients in a function field. That work, however, remains unpublished. Igusa [5] showed that for a pre- 
homogeneous vector space that has locally only one nonsingular orbit the local functional equation coefficient is essentially the $\Gamma$-function of Tate's thesis [13]. Recently Muller [6], following the work of Rallis and Schiffmann [7], showed how to compute the local functional equation coefficients for zeta functions associated with prehomogeneous vector spaces of commutative parabolic type. Finally, in [9] F. Sato computed the local functional equation coefficients for several prehomogeneous vector spaces. Our local functional equation is a particular case of one of the functional equations of Sato ([9], Theorem 3.6 with $Q(x)=x_{1} x_{3}-x_{2}^{2}$, $\omega^{(1)}=1$ and $\omega^{(2)}=\omega_{s}$ )

Sato expresses his coefficients as linear combinations of products of Gauss sums of quadratic characters on $K_{\nu}^{\times}$and Tate $\Gamma$-functions. This elegant formulation certainly sheds much better light on the nature of the functional equation coefficients. Moreover, it is more general than mine, and it applies to ramified as well as unramified quasicharacters. Thus my results in Section 2 of this paper can not strictly speaking be considered new. Nevertheless, I chose to include then in this paper because my methods are different from Sato's and because my calculation is more explicit than his. The calculation of the remainder term $T_{\mathrm{x}_{s}}(s)$ is completely new and can be considered the original feature of this paper.

0.4. Let $H(n)$ denote the class number of positive definite integral binary quadratic forms of discriminant $-n$ where the forms equivalent to $a\left(u^{2}+\nu^{2}\right)$ and $a\left(u^{2}+u v+v^{2}\right)$ are counted with multiplicities $\frac{1}{2}$ and $\frac{1}{3}$ respectively. Following Cohen [1], let $H(0)=-\frac{1}{12}$, and let

$$
\mathscr{H}_{1}(z)=\sum_{n=0}^{\infty} H(n) e^{2 \pi i n z}, \quad \operatorname{Im}(z)>0 .
$$

Zagier [17] discovered a remarkable fact: let

$$
\mathscr{G}(z)=\mathscr{H}_{1}(z)+\frac{1}{16 \pi \sqrt{y}} \sum_{f=-\infty}^{\infty} \alpha\left(f^{2} y\right) e^{-2 \pi i f^{2} z}, \quad z=x+i y
$$

where $\alpha(t)=\int_{1}^{\infty} e^{-4 \pi u t} u^{-\frac{3}{2}} d u$. Then $\mathscr{G}(z)$ transforms under $\Gamma_{0}(4)$ as a modular form of weight $3 / 2$. As a corollary,

$$
\mathscr{H}_{1}\left(\frac{a z+b}{c z+d}\right)=\left(\frac{c}{d}\right)\left(\frac{-4}{d}\right)^{\frac{1}{2}}(c z+d)^{\frac{3}{2}} \mathscr{H}_{1}(z)-\frac{1+i}{16 \pi} \int_{-\frac{a}{c}}^{i \infty} \frac{\theta(t) d t}{(t+z)^{\frac{3}{2}}}
$$


for any $\left(\begin{array}{ll}a & b \\ c & d\end{array}\right) \in \Gamma_{0}(4)$.

The functional equations of Shintani $(0.1)$ for $\xi_{-}(s)$ and Zagier (0.5) are, in fact, two reflections of the same phenomenon though the connection between the two remains somewhat unclear. In the last section of this paper we explore the connection between zeta functions associated with the space of binary quadratic forms and modular forms of weight $3 / 2$. More precisely, we give a method for constructing Dirichlet series $\xi(s)=\sum_{n} a(n) \lambda_{n}^{s}$, where $\lambda_{n}$ are rational numbers with bounded denominators, that satisfy

$$
\left(\frac{\pi}{2}\right)^{s-\frac{3}{2}} \Gamma\left(\frac{3}{2}-s\right) \xi\left(\frac{3}{2}-s\right)=\left(\frac{\pi}{2}\right)^{-s} \Gamma(s) \xi(s)
$$

and write down explicitly one of these series. Not surprisingly it turns out to be the Mellin transform of a linear combination of $\mathscr{G}(m z+l / a)$ for some integers $m$, $l$ and $a$.

Unfortunately, we can not yet deduce the functional equation of Zagier from the theory of zeta functions associated with the space of binary quadratic forms. However, the tools for doing so already exist. In [8] Saito showed how to twist zeta functions associated with the space of binary quadratic forms by multiplicative characters modulo $p$. This combined with the Weil criterion for forms of half integral weight (see [11], p. 481) ought to lead to the functional equation of Zagier or a result very close to it.

0.5. This paper is organized as follows: Section 1 contains a summary of relevant facts from [3] without proofs. This is done in order to make the paper self-contained. In Section 2 we compute the local functional equation coefficients $\Gamma_{\mathrm{x}_{\nu} \mathrm{y}_{\nu}}(s)$. Section 3 is devoted to computing the remainder term $T_{\mathrm{x}_{s}}(s)$. Finally, in Section 4 we draw parallels between zeta functions associated with the space of binary quadratic forms and modular forms of weight $3 / 2$.

0.6. Acknowledgements. This work was prepared while the author was a guest at Sonderforschungsbereich-170, Mathematisches Institut, Göttingen and at the Technion, Israel Institute of Technology. The author wishes to thank SFB-170 and the Technion for their hospitality and SFB-170 for its generous support.

\section{Zeta functions, associated with the space of binary quadratic forms}

\subsection{The space of binary quadratic forms}


Let $V$ be the 3 -dimensional affine space. We identify $V$ with the space of quadratic forms via the correspondence:

$$
x=\left(x_{1}, x_{2}, x_{3}\right) \in V \leftrightarrow F_{x}(u, v)=x_{1} u^{2}+x_{2} u v+x_{3} v^{2} .
$$

The group $G l_{2}$ acts on $V$ by the linear change of variables. This action, however, does not allow for scalar multiplication (though it does allow scalar multiplication by squares). Therefore set $G=G l_{1} \times G l_{2}$. Explicitly, the action of $G$ on $V$ is given by:

$$
F_{g \cdot x}(u, v)=t F_{x}(a u+c v, b u+d v)
$$

for $g=\left(t,\left(\begin{array}{ll}a & b \\ c & d\end{array}\right)\right) \in G$ and $x \in V$.

For $x \in V$ let $P(x)$ denote the discriminant of $x$ :

$$
P(x)=x_{2}^{2}-4 x_{1} x_{3}
$$

For $g=\left(t,\left(\begin{array}{ll}a & b \\ c & d\end{array}\right)\right) \in G$, set $\chi(g)=(t(a d-b c))^{2}$. Then $P(g \cdot x)=\chi(g) P(x)$.

We call a form $x$ non-singular if $P(x) \neq 0$ and singular otherwise. It is easy to see that two non-singular forms in $V_{K}$ are $G_{K}$-equivalent if and only if their splitting fields over $K$ are the same. Thus non-singular $G_{K}$-orbits in $V_{K}$ are in one-to-one correspondense with extensions of $K$ of degree less or equal to 2. In particular, if $K$ is algebraically closed, $G_{K}$ has a Zariski-open orbit $V_{K}^{\prime}=\{x \in$ $\left.V_{K}: P(x) \neq 0\right\}$ in $V_{K}$. Thus the triple $(G, \varrho, V)$ is a prehomogeneous vector space in the sense of [10].

Let $K_{x}$ denote the splitting field of the form $x \in V_{K}$ over $K$. Define $V_{K}^{\prime \prime}=$ $\left\{x \in V_{K}^{\prime}:\left[K_{x}: K\right]=2\right\}$. The stabilizer $G_{x}$ of $x \in V_{K}^{\prime \prime}$ has a rather interesting property. Let $G_{x}^{0}$ be the connected component of $G_{x}$. Then $\left|G_{x} / G_{x}^{0}\right|=2$ and $G_{x}^{0} \cong R_{K_{x} / K}\left(G_{m}\right)$ where $G_{m}$ is the multiplicative group and $R_{K_{x} / K}$ denotes the base restriction from $K_{x}$ to $K$. For details we refer the reader to [3], Section 1.

Now let $K$ be an algebraic number field. For $\nu \in M(K)$ let $K_{\nu}$ denote the completion of $K$ at $\nu$. If $K_{\nu}$ is non-archimedean, let $O_{\nu}$ denote the ring of integers in $K_{\nu}$.

Let $A=\Pi_{\nu \in M(K)}^{\prime} K_{\nu}$ denote the ring of adeles of $K$ and $A^{\times}$its group of ideles. Endowed with the restricted product topologies, $A$ and $A^{\times}$become a locally compact topological ring and group respectively. The group $A^{\times}$is endowed with the adelic absolute value ||$_{A}$. The field $K$, identified with a subset of $A$ via the diagonal embedding, forms a lattice in $A$.

Let $V_{A}$ denote the space of binary quadratic forms with coefficients in $A$. 
Then $V_{K}$ forms a $G_{K}$-invariant lattice in $V_{A}$. We note that both the sets $V_{K}^{\prime}$ and $V_{K}^{\prime \prime}$ are $G_{K}$-invariant subsets of $V_{K}$.

The action of $G$ on $V$ defines a representation $\varrho: G \rightarrow G l(V)$ defined over $K$. The kernel of $\varrho$ is a one-dimensional torus $T_{\varrho}$ in the center of $G, T_{\varrho}=$ $\left\{\left(t^{-2},\left(\begin{array}{cc}t & 0 \\ 0 & t\end{array}\right)\right)\right\}$. The image of $\varrho, H$, is a closed reductive subgroup of $G l(V)$ of semisimple rank 1 and dimension 4. Define $H_{A}$ following [14]. Then $H_{A}$ is a subgroup of $G l\left(V_{A}\right)$, and $H_{K}$ is a discrete subgroup of $H_{A}$.

Since in much of the paper we will be engaged in calculations that involve integration with respect to local and global invariant measures on $H$, we are going to normalize our measures here once and for all.

The global measure $d h$ on $H_{A}$ is given as follows. Let $d u$ and $d^{\times} t$ denote invariant measures on $A$ and $A^{\times} \cdot d^{\times} t=\frac{d|t|_{A}}{|t|_{A}} d^{1} t$ where $d^{1} t$ is a multiplicative invariant measure on $A^{1}=\left\{t \in A^{\times}:|t|_{A}=1\right\}$. We normalize $d u$ and $d^{\times} t$ by setting $\int_{A / K} d u=1$ and $\int_{A^{1} / K^{\times}} d^{1} t=1$. As in [3], let $n(u)=\left(1,\left(\begin{array}{ll}1 & 0 \\ u & 1\end{array}\right)\right), d\left(t, t_{1}\right)$ $=\left(t,\left(\begin{array}{cc}t_{1} & 0 \\ 0 & t_{1}\end{array}\right)\right)$, and $a(\tau)=\left(1,\left(\begin{array}{ll}1 & 0 \\ 0 & \tau\end{array}\right)\right)$. The group $G_{A}$ has an Iwasawa decomposition $G_{A}=\mathscr{K} B_{A}$ where $\mathscr{K}$ is the standard maximal compact subgroup of $G_{A}$ and $B$ a Borel subgroup of $G$. More specifically, $\mathscr{K}=\Pi_{\nu \in M(K)} \mathscr{K}_{\nu}$ where $\mathscr{K}_{\nu}=$ $G_{O_{\nu}}$ is $\nu$ is non-archimedean, $\mathscr{K}_{\nu}=\left( \pm 1, O_{2}(\mathbf{R})\right)$ if $K_{\nu}=\mathbf{R}$ and $K_{\nu}=\left(1, U_{2}(\mathbf{C})\right)$ if $K_{\nu}=\mathbf{C}$, and $B=\left\{\left(t,\left(\begin{array}{ll}t_{1} & 0 \\ u & t_{2}\end{array}\right)\right)\right\}$.

Define the measure $d g$ on $G_{A}$ by $d g=d \kappa d b$. Every element of $B_{A}$ can be written uniquely as $b=d\left(t, t_{1}\right) n(u) a(\tau)$ where $u \in A$ and $t, t_{1}, \tau \in A^{\times}$, and one easily checks that $d b=d^{\times} t d^{\times} t_{1} d u d^{\times} \tau$ is a right-invariant measure on $B_{A}$. Finally, normalize $d \kappa$ by setting $\int_{\mathscr{K}} d \kappa=1$. We now have a normalized Haar measure $d g$ on $G_{A}$.

$H \cong G / T_{\varrho}$ where $T_{\varrho}=\left\{d\left(t_{1}^{-2}, t_{1}\right) \in G\right\}$. Define $d h$ by setting $d g=d^{\times} t_{1} d h$. More explicitly, write $h=\varrho(\kappa d(t, 1) n(u) a(\tau))$. Then $d h=d \kappa d^{\times} t d u d^{\times} \tau$.

Denote by $d x_{\nu}$ the additive Haar measure on $K_{\nu}$ normalized as follows: $d x_{\nu}$ is the usual measure if $K_{\nu}=\mathbf{R}, d x_{\nu}=\left|d x_{\nu} \wedge d \bar{x}_{\nu}\right|$ if $K_{\nu}=\mathbf{C}$, and $\int_{O_{\nu}} d x_{\nu}=1$ if $K_{\nu}$ is non-archimedean. Set the multiplicative Haar neasure $d^{\times} x_{\nu}$ on $K_{\nu}^{\times}$to be $\frac{d x_{\nu}}{\left|x_{\nu}\right|_{\nu}}$ 
if $K_{\nu}=\mathbf{R}$ or $\mathbf{C}$, and the measure, normalized by $\int_{O_{\nu}^{\times}} d^{\times} x_{\nu}=1$, if $K_{\nu}$ is a non-archimedean local field. We note that $d x=D_{K}^{-\frac{1}{2}} \Pi_{\nu \in M(K)} d x_{\nu}$ and $d^{\times} x=$ $\rho_{K}^{-1} \prod_{\nu \in M(K)} d^{\times} x_{\nu}$.

Set $d h_{\nu}=d \kappa_{\nu} d^{\times} t_{\nu} d^{\times} \tau_{\nu} d u_{\nu}$ where $h_{\nu}=\varrho\left(\kappa_{\nu} d\left(t_{\nu}, 1\right) n\left(u_{\nu}\right) a\left(\tau_{\nu}\right)\right)$. Clearly, $d h=$ $D_{K}^{-\frac{1}{2}} \rho_{K}^{-2} \Pi_{\nu \in M(K)} d h_{\nu}$.

\subsection{The global zeta function}

Let $\&\left(V_{A}\right)$ denote the set of Schwartz-Bruhat functions on $V_{A}$. For $\Phi \in$ $\&\left(V_{A}\right)$ define

$$
Z(s, \Phi)=\int_{H_{A} / H_{K}}|\chi(h)|_{A}^{s} \sum_{x \in V_{K}^{\prime \prime}} \Phi(h \cdot x) d h
$$

where $d h$ is a left invariant Haar measure on $H_{A}$. This is the global zeta function associated with the space of binary quadratic forms.

Let \langle\rangle$: A \rightarrow \mathbf{C}^{\times}$be a non-trivial additive character on $A$ that is trivial on $K$. Define a bilinear form [,] on $V_{A}$ :

$$
[x, y]=x_{1} y_{3}-\frac{1}{2} x_{2} y_{2}+x_{3} y_{1} .
$$

Set $d x=d x_{1} d x_{2} d x_{3}$. For $\Phi \in \&\left(V_{A}\right)$, define the Fourier transform $\Phi^{*}$ of $\Phi$ by

$$
\Phi^{*}(y)=\int_{V_{A}} \Phi(x)\langle[x, y]\rangle d x
$$

The properties of the global zeta function $Z(s, \Phi)$ are summarized in the following theorem due to A. Yukie. For a proof we refer the reader to [16] or [3].

THEOREM 1.1. i) For any $\Phi \in \mathscr{S}\left(V_{A}\right)$, the integral defining $Z(s, \Phi)$ converges absolutely and locally uniformly in the half-plane $\operatorname{Re}(s)>\frac{3}{2}$.

ii) $Z(s, \Phi)$ can be analytically continued to a meromorphic function in the entire complex plane.

iii) $Z(s, \Phi)$ satisfies a functional equation

$$
Z\left(\frac{3}{2}-s, \Phi\right)=Z\left(s, \Phi^{*}\right)+\left(T\left(2 s, \Phi^{*}\right)-T(3-2 s, \Phi)\right) .
$$

The distribution $T(s, \Phi)$ is rather curious. Let 


$$
\alpha(u)=\prod_{\nu \in M_{\mathbf{R}}(K)}\left(1+\left|u_{\nu}\right|_{\nu}^{2}\right)^{\frac{1}{2}} \prod_{\nu \in M_{\mathbf{C}}(K)}\left(1+\left|u_{\nu}\right|_{\nu}\right) \prod_{\nu \in M_{0}(K)} \sup \left(1,\left|u_{\nu}\right|_{\nu}\right)
$$

Define

$$
T(s, w, \Psi)=\int_{A^{\times}} \int_{A}|t|_{A}^{s} \Psi(t, t u) \alpha(u)^{w} d u d^{\times} t
$$

$T(s, w, \Psi)$ is holomorphic in the region $\operatorname{Re}(s)>1, \operatorname{Re}(s-w)>2$ and can be continued meromorphically to the entire space $\mathbf{C}^{2}$.

For $\Phi \in \mathscr{S}\left(V_{A}\right)$ set $M \Phi(x)=\int_{\mathscr{K}} \Phi(\kappa \cdot x) d \kappa$. Define the truncating distribution $T_{2} \Phi$ by setting $T_{2} \Phi(t, u)=\Phi(0, t, u)$. Then

$$
T(s, \Phi)=\left.\frac{d}{d w} T\left(s, w, T_{2}(M \Phi)\right)\right|_{w=0}
$$

Remark. 1. The poles of $Z(s, \Phi)$ occur at $s=\frac{3}{2}$ (at most simple), at $s=1$ (at most double), $s=\frac{1}{2}$ (at most double), $s=0$ (at most simple), and at $s=\frac{-n}{2}$, $n=1,2, \ldots[16]$ and [3] contain explicit formulae for residues at these poles. This information, however, is irrelevant for the purposes of this paper.

Remark. 2. The adelic zeta function, associated with a prehomogeneous vector space $(H, \rho, V)$, is usually defined as $Z(s, \Phi)=\int_{H_{A} / H_{K}}|\chi(h)|_{A}^{s} \Sigma_{x \in V_{K}^{\prime}}$ $\Phi(h \cdot x) d h$ where $V_{K}^{\prime}=\left\{x \in V_{K}: P(x) \neq 0\right\}$. Normally, such a zeta function satisfies a functional equation without a remainder (see [10]). For reasons of convergence we had to restrict our sum under the integral sign to $x \in V_{K}^{\prime \prime}$. The points in $V_{K}^{\prime}-V_{K}^{\prime \prime}$ that we have omitted lead to the remainder term $\left(T\left(2 s, \Phi^{*}\right)\right.$ $T(3-2 s, \Phi))$ in the functional equation of $Z(s, \Phi)$.

\subsection{The local zeta functions}

Let $K_{\nu}$ be a completion of the field $K$. Since $H_{K_{\nu}}$-orbits in $V_{K_{\nu}}^{\prime}$ are in one-to-one correspondence with extensions of $K_{\nu}$ of degree less or equal to 2 , their number is finite. For each $H_{K_{\nu}}$-orbit $V_{i} \in V_{K_{\nu}}^{\prime}$ define

$$
Z_{V_{i}}\left(s, \Phi_{\nu}\right)=\int_{V_{i}}\left|P\left(y_{\nu}\right)\right|_{\nu}^{s} \Phi\left(y_{\nu}\right) \frac{d y_{\nu}}{\left|P\left(y_{\nu}\right)\right|_{\nu}^{\frac{3}{2}}} .
$$

$Z_{V_{i}}\left(s, \Phi_{\nu}\right)$ is called a local zeta function associated with the space of binary 
quadratic forms.

Let \langle\rangle$_{\nu}$ be an additive character on $K_{\nu}$ given as follows: $\langle x\rangle_{\nu}=e^{2 \pi \imath x}$ if $K_{\nu}=\mathbf{R},\langle x\rangle_{\nu}=e^{4 \pi i \operatorname{Re}(x)}$ if $K_{\nu}=\mathbf{C}$, and \langle\rangle$_{\nu}$ of order 0 (i.e. $\langle x\rangle_{\nu}=1$ if and only if $x \in O_{\nu}$ ) if $K_{\nu}$ is non-archimedean. Define the local Fourier transform

$$
\Phi_{\nu}^{*}\left(y_{\nu}\right)=\int_{V_{K_{\nu}}} \Phi_{\nu}\left(x_{\nu}\right)\left\langle\left[x_{\nu}, y_{\nu}\right]\right\rangle_{\nu} d x_{\nu}
$$

The local zeta function $Z_{V_{i}}\left(s, \Phi_{\nu}\right)$ has the following invariance property: for $h_{\nu} \in H_{K_{\nu}}$ let $\left(h_{\nu} \cdot \Phi_{\nu}\right)\left(x_{\nu}\right)=\Phi_{\nu}\left(h_{\nu}^{-1} \cdot x_{\nu}\right)$. Then

$$
Z_{V_{i}}\left(s, h_{\nu} \cdot \Phi_{\nu}\right)=\left|\chi\left(h_{\nu}\right)\right|_{\nu}^{s} Z_{V_{i}}\left(s, \Phi_{\nu}\right) .
$$

From uniqueness of the Haar measure it follows at once that any distribution with support in $V_{i}$ that has the invariance property (1.7) is a constant multiple of $Z_{V_{i}}\left(s, \Phi_{\nu}\right)$. Therefore for any $\Phi_{\nu}$ with support in $V_{K_{\nu}}^{\prime}$

$$
Z_{V_{j}}\left(s, \Phi_{\nu}^{*}\right)=\sum_{j} \gamma_{i j}^{\nu}(s) Z_{V_{i}}\left(\frac{3}{2}-s, \Phi_{\nu}\right)
$$

In fact, by the same argument as in [4], Section 3, (1.8) holds for all $\Phi_{\nu} \in \mathscr{S}\left(V_{K_{\nu}}\right)$.

Let $x_{\nu} \in V^{\prime}\left(K_{\nu}\right)$. Denote by $V_{x_{\nu}}$ the $H_{K_{\nu}}$-orbit of $x_{\nu}$ in $V_{K_{\nu}}$. Let $H_{x_{\nu}}^{0}$ denote the connected component of the stabilizer of $x_{\nu}$ in $H$. The map $H_{K_{\nu}} /\left(H_{x_{\nu}}^{0}\right)_{K_{\nu}} \rightarrow V_{x_{\nu}}$, $h_{\nu} \rightarrow h_{\nu} \cdot x_{\nu}$, gives a double covering of $V_{x_{\nu}}$. Set the measure $d_{x_{\nu}}^{\prime} h_{\nu}$ on $H_{K_{\nu}} /\left(H_{x_{\nu}}^{0}\right)_{K_{\nu}}$ locally equal to $b_{x_{\nu}} \frac{d y_{\nu}}{\left|P\left(y_{\nu}\right)\right|_{\nu}^{\frac{3}{2}}}$ where $b_{x_{\nu}}$ is a constant whose value depends only on the orbit $V_{x_{\nu}}$ and not on $x_{\nu}$ itself. It is easy to see that $d_{x_{\nu}}^{\prime} h_{\nu}$ is an $H_{K_{\nu}}$-left invariant measure on $H_{K_{\nu}} /\left(H_{x_{\nu}}^{0}\right)_{K_{\nu}}$.

The values of $b_{x_{\nu}}$ are given in Propositions 4.2-4.4 of [3]; the reason for introducing $b_{x_{\nu}}$ in the measure will become apparent in the next section.

Define

$$
Z_{x_{\nu}}\left(s, \Phi_{\nu}\right)=\int_{H_{K_{\nu}} /\left(H_{x_{\nu}}^{0}\right)_{K_{\nu}}}\left|\chi\left(h_{\nu}\right)\right|_{\nu}^{s} \Phi_{\nu}\left(h_{\nu} \cdot x_{\nu}\right) d_{x_{\nu}}^{\prime} h_{\nu}
$$

Then

$$
Z_{x_{\nu}}\left(s, \Phi_{\nu}\right)=b_{x_{\nu}}\left|P\left(x_{\nu}\right)\right|_{\nu}^{-s} Z_{V_{x_{\nu}}}\left(s, \Phi_{\nu}\right) .
$$

For each orbit $V_{x_{\nu}}$ choose a standard orbital representative $x_{\nu}$ as follows. If the splitting field $K_{x_{\nu}}=K_{\nu}$ set $F_{\mathrm{x}_{\nu}}(u, v)=u v$. If $\left[K_{x_{\nu}}: K_{\nu}\right]=2$ and $K_{\nu}$ is non-archimedean, set $F_{\mathrm{x}_{\nu}}(u, v)=(u+\theta v)\left(u+\theta^{\prime} v\right)$ where $O_{x_{\nu}}=O_{\nu}[\theta]$. Here $O_{x_{\nu}}$ stands for the ring of integers in $K_{x_{\nu}}$ and $\theta^{\prime}$ denotes the Galois conjugate of $\theta$ 
over $K_{\nu}$. Finally, if $K_{\nu}=\mathbf{R}$ and $K_{x_{\nu}}=\mathbf{C}$, set $F_{\mathbf{x}_{\nu}}(u, v)=\frac{1}{2}\left(u^{2}+v^{2}\right)$. Note that $\left|P\left(\mathrm{x}_{\nu}\right)\right|_{\nu}=D_{K_{x_{\nu}} / K_{\nu}}^{-1}$ where $D_{K_{x_{\nu}} / K_{\nu}}$ is the norm of the discriminant of $K_{x_{\nu}}$ over $K_{\nu}$.

In all computations that follow we will replace $Z_{V_{x_{\nu}}}\left(s, \Phi_{\nu}\right)$ by $Z_{\mathrm{x}_{\nu}}\left(s, \Phi_{\nu}\right)$. Therefore we will rewrite (1.8) as

$$
Z_{\mathrm{y}_{\nu}}\left(s, \Phi_{\nu}^{*}\right)=\sum_{\mathrm{y}_{\nu}} \Gamma_{\mathrm{x}_{\nu} \mathrm{y}_{\nu}}(s) Z_{\mathrm{x}_{\nu}}\left(\frac{3}{2}-s, \Phi_{\nu}\right)
$$

\subsection{An adelic synthesis}

The zeta function $Z(s, \Phi)$ has no Euler product; however, it is fairly easy to decompose it into the sum of integrals that do. Since the integral (1.1) defining $Z(s, \Phi)$ converges absolutely for $\operatorname{Re}(s)>\frac{3}{2}$, we can interchange the order of summation and integration. Then

$$
Z(s, \Phi)=\frac{1}{2} \sum_{x \in H_{K} \backslash V_{K}^{\prime \prime}} \int_{H_{A^{\prime}}\left(H_{x}^{0}\right)_{K}}|\chi(h)|_{A}^{s} \Phi(h \cdot x) d h .
$$

Note that $H_{K}$-orbits in $V_{K}^{\prime \prime}$ are in one-to-one correspondence with quadratic extensions of $K$. Thus the sum in (1.12) is actually a sum over the quadratic extensions of $K$.

Each of the integrals in (1.12) can be written as

$$
b_{x} \mu(x) \int_{H_{A} /\left(H_{x}^{0}\right)_{A}}\left|\chi\left(h^{\prime}\right)\right|_{A}^{s} \Phi\left(h^{\prime} \cdot x\right) d_{x}^{\prime} h^{\prime}
$$

where

$$
\mu(x)=\int_{\left(H_{x}^{0}\right)_{A} /\left(H_{x}^{0}\right)_{K}} d_{x}^{\prime \prime} h^{\prime \prime}
$$

Here $d_{x}^{\prime} h^{\prime}$ and $d_{x}^{\prime \prime} h^{\prime \prime}$ are the measures on $H_{A} /\left(H_{x}^{0}\right)_{A}$ and $\left(H_{x}^{0}\right)_{A} /\left(H_{x}^{0}\right)_{K}$ respectively, and $b_{x}$ is given by $d h=b_{x} d_{x}^{\prime} h^{\prime} d_{x}^{\prime \prime} h^{\prime \prime}$.

Since $G_{x}^{0} \cong R_{K_{x} / K}\left(G_{m}\right)$ it is hardly surprizing that for an appropriate choice of $d_{x}^{\prime \prime} h^{\prime \prime} \mu(x)$ is essentially $\operatorname{Vol}\left(A_{K_{x}}^{1} / K_{x}^{\times}\right)=\rho_{K_{x}}$. In fact, if we choose $d_{x}^{\prime \prime} h^{\prime \prime}=$ $\Pi_{\nu} d^{\prime \prime} h_{\nu}^{\prime \prime}$ as in [3], $\mu(x)=\frac{2 \rho_{K_{x}}}{\rho_{K}}$.

Set $d_{x}^{\prime} h^{\prime}=\Pi_{\nu \in M(K)} d_{x}^{\prime} h_{\nu}^{\prime}$ where $d_{x}^{\prime} h_{\nu}^{\prime}$ are the local measures, described in the previous section. The constants $b_{x_{\nu}}$ in [3] were chosen so that $d h_{\nu}=d_{x_{\nu}}^{\prime} h_{\nu}^{\prime} d_{x_{\nu}}^{\prime \prime} h_{\nu}^{\prime \prime}$. Therefore $d h=D_{K}^{-\frac{1}{2}} \rho_{K}^{-2} d_{x}^{\prime} h^{\prime} d_{x}^{\prime \prime} h^{\prime \prime}$. 
Let $\Phi=\Pi_{\nu \in M(K)} \Phi_{\nu}$ be a Schwartz-Bruhat function on $V_{A}$. Then the integral in (1.13) has an Euler product $\Pi_{\nu \in M(K)} Z_{x}\left(s, \Phi_{\nu}\right)$. For each $\nu \in M(K)$ let $\mathrm{x}_{\nu}$ denote the standard orbital representative of $x$ in $V_{K_{\nu}}$. In view of (1.10), (1.13) equals

$$
\prod_{\nu \in M(K)}\left|\frac{P\left(\mathrm{x}_{\nu}\right)}{P(x)}\right|_{\nu}^{s} \prod_{\nu \in M(K)} Z_{\mathrm{x}_{\nu}}\left(s, \Phi_{\nu}\right)=D_{K_{x} / K}^{-s} \prod_{\nu \in M(K)} Z_{\mathrm{x}_{\nu}}\left(s, \Phi_{\nu}\right) .
$$

The orbit of $x$ over $K_{\nu}$ depends only on the splitting type of the field $K_{x}$ over $K_{\nu}$. Consequently we can think of $\mathrm{x}_{\nu}$ as the $\nu$-splitting signature of the quadratic field $K_{x}$. Similarly, we can think of $\mathrm{x}_{S}=\left(\mathrm{x}_{\nu}\right)_{\nu \in S}$ as the $S$-splitting signature of $K_{x}$. As in the Introduction, we will write $K_{x} \sim \mathrm{x}_{S}$ to indicate that a quadratic field $K_{x}$ has the $S$-splitting signature $\mathrm{x}_{S}$.

For any $\Phi=\Pi_{\nu \in M(K)} \Phi_{\nu} \in \mathscr{S}\left(V_{A}\right)$ there exists a finite set $S$ of places of $K$ such that $\forall \nu \notin S \Phi_{\nu}=\Phi_{0, \nu}$ where $\Phi_{0, \nu}$ is the characteristic function of $V_{O_{\nu}}$. Consequently set

$$
\eta_{x, S}(s)=\prod_{\nu \notin S} Z_{\mathrm{x}_{\nu}}\left(s, \Phi_{0, \nu}\right)
$$

and let

$$
\xi_{\mathrm{x}_{s}}(s)=\sum_{K_{x} \sim \mathrm{x}_{s}} \frac{\rho_{K_{x}}}{D_{K_{x} / K}^{s}} \eta_{x, S}(s) .
$$

Finally, let

$$
Z_{\mathrm{x}_{S}}(s, \Phi)=\prod_{\nu \in S} Z_{\mathrm{x}_{\nu}}\left(s, \Phi_{\nu}\right)
$$

Then

$$
Z(s, \Phi)=D_{K}^{-\frac{1}{2}} \rho_{K}^{-3} \sum_{\mathrm{x}_{s}} Z_{\mathrm{x}_{s}}(s, \Phi) \xi_{\mathrm{x}_{s}}(s) .
$$

The additive character $\langle>$ on $A / K$ decomposes as a product of local additive characters. The order of its local character at $\nu$, however, is not 0 ; it is $n_{\nu}=\operatorname{ord}_{\nu}$ $\mathscr{D}_{\nu}$ where $\mathscr{D}_{\nu}$ is the different of $K$ at $\nu$. Therefore $\langle x\rangle=\Pi_{\nu \in M(K)}\left\langle\pi_{\nu}^{n_{\nu}} x_{\nu}\right\rangle_{\nu}$. Hence the Fourier transform $\Phi^{*}(y)=D_{K}^{-\frac{3}{2}} \Pi_{\nu \in M(K)} \Phi_{\nu}^{*}\left(\pi_{\nu}^{n_{\nu}} y_{\nu}\right)$.

Set

$$
\eta_{x, S}^{*}(s)=\prod_{\nu \notin S} Z_{\mathrm{x}_{\nu}}\left(s, \Phi_{0, \nu}^{*}\right)
$$

and 


$$
\xi_{\mathrm{x}_{S}}^{*}(s)=\sum_{K_{x} \sim \mathrm{x}_{S}} \frac{\rho_{K_{x}}}{D_{K_{x} / K}^{s}} \eta_{x, S}^{*}(s) .
$$

Then

$$
Z\left(s, \Phi^{*}\right)=D_{K}^{-\frac{1}{2}} \rho_{K}^{-3} D_{K}^{-\frac{3}{2}} \sum_{\mathrm{x}_{s}} Z_{\mathrm{x}_{s}}\left(s, \Phi^{*}\right) \xi_{\mathrm{x}_{s}}^{*}(s) .
$$

Combining expansions (1.18) and (1.21) with the functional equations (1.2) and (1.11) we obtain the following theorem:

THEOREM 1.2. The Dirichlet series $\xi_{\mathrm{x}_{s}}(s), \xi_{\mathrm{x}_{s}}^{*}(s)$ satisfy the functional equation

$$
\xi_{\mathrm{x}_{s}}\left(\frac{3}{2}-s\right)=D_{K}^{2 s-\frac{3}{2}} \sum_{\mathrm{y}_{s}} \Gamma_{\mathrm{x}_{s} \mathrm{y}_{s}}(s) \xi_{\mathrm{y}_{s}}^{*}(s)+T_{\mathrm{x}_{s}}(s) .
$$

For $\mathrm{x}_{S}=\left(\mathrm{x}_{\nu}\right)_{\nu \in S}$ and $\mathrm{y}_{S}=\left(\mathrm{y}_{\nu}\right)_{\nu \in S} \Gamma_{\mathrm{x}_{S} \mathrm{y}_{S}}(s)=\Pi_{\nu \in S} \Gamma_{\mathrm{x}_{\nu} \mathrm{y}_{\nu}}(s)$ where $\Gamma_{\mathrm{x}_{\nu} \mathrm{y}_{\nu}}(s)$ are the functional equation coefficients in equation (1.11)

The values of $Z_{\mathrm{x}_{\nu}}\left(s, \Phi_{0, \nu}\right)$ can be found in Proposition 4.1 of [3]. We restate this proposition here without a proof.

Proposition 1.3. Let $\Phi_{0, \nu}$ be the characteristic function of $V_{O_{\nu}}$. Then

$$
Z_{\mathrm{x}_{\nu}}\left(s, \Phi_{0, \nu}\right)= \begin{cases}\frac{1}{1-q_{\nu}^{1-2 s}} & \text { if } K_{\mathrm{x}_{\nu}}=K_{\nu} ; \\ \frac{1+q_{\nu}^{-2 s}}{\left(1-q_{\nu}^{-2 s}\right)\left(1-q_{\nu}^{1-2 s}\right)} & \text { if }\left[K_{\mathrm{x}_{\nu}}: K_{\nu}\right]=2, \text { unramified } ; \\ \frac{1}{\left(1-q_{\nu}^{-2 s}\right)\left(1-q_{\nu}^{1-2 s}\right)} & \text { if }\left[K_{\mathrm{x}_{\nu}}: K_{\nu}\right]=2, \text { ramified, }\end{cases}
$$

and

$$
\eta_{x, S}(s)=\frac{\zeta_{K, S}(2 s-1) \zeta_{K, S}(2 s)^{2}}{\zeta_{K_{x}, S}(2 s)}
$$

where $\zeta_{K, S}(s)=\Pi_{\nu \notin S}\left(1-q_{\nu}^{-s}\right)^{-1}$ is a truncated Dedekind zeta function.

The last assertion of the proposition follows easily from (1.15) and the values of $Z_{\mathrm{x}_{\nu}}\left(s, \Phi_{0, \nu}\right)$.

If $\nu \times 2, \Phi_{0, \nu}^{*}=\Phi_{0, \nu}$. If $\nu \mid 2, \Phi_{0, \nu}^{*}$ is the characteristic function of $O_{\nu} \times 2 O_{\nu}$ $\times O_{\nu}$. The values of $Z_{\mathbf{x}_{\nu}}\left(s, \Phi_{2, \nu}\right)$ are rather easy to obtain from Proposition 1.3. 
Note that $O_{\nu} \times 2 O_{\nu} \times O_{\nu}=\left\{x_{\nu} \in V_{O_{\nu}}:\left|P\left(x_{\nu}\right)\right|_{\nu} \leq|4|_{\nu}\right\}$. Writing $x_{\nu}=h_{\nu} \cdot \mathrm{x}_{\nu}$ we see that $\left|\chi\left(h_{\nu}\right)\right|_{\nu}^{2} \leq \frac{|4|_{\nu}}{\left|P\left(\mathrm{x}_{\nu}\right)\right|_{\nu}}$. Therefore in the integral defining $Z_{\mathrm{x}_{\nu}}\left(s, \Phi_{0, \nu}\right)$ we only need to integrate over only those $h_{\nu}$ with $\left|\chi\left(h_{\nu}\right)\right|_{\nu} \leq \frac{|2|_{\nu}}{\left|P\left(\mathrm{x}_{\nu}\right)\right|_{\nu}^{\frac{1}{2}}}$. By partial fractions, we obtain

(1.22) $Z_{\mathrm{x}_{\nu}}\left(s, \Phi_{0, \nu}^{*}\right)=$

$$
\begin{cases}\frac{|2|_{\nu}^{2 s-1}}{1-q_{\nu}^{1-2 s}} & \text { if } K_{\mathrm{x}_{\nu}}=K_{\nu} \\ \frac{1}{q_{\nu}-1}\left(\left(q_{\nu}+1\right) \frac{|2|_{\nu}^{2 s-1}}{1-q_{\nu}^{1-2 s}}-2 \frac{|2|_{\nu}^{2 s}}{1-q_{\nu}^{-2 s}}\right) & \text { if }\left[K_{\mathrm{x}_{\nu}}: K_{\nu}\right]=2 \text {, unramified. }\end{cases}
$$

If $K_{\mathrm{x}_{\nu}}$ is ramified over $K_{\nu}$,

$$
\begin{aligned}
& Z_{\mathrm{x}_{\nu}}\left(s, \Phi_{0, \nu}^{*}\right)= \\
& \begin{cases}\frac{1}{q_{\nu}-1}\left(q_{\nu} \frac{|2|_{\nu}^{2 s-1}\left|P\left(\mathrm{x}_{\nu}\right)\right|_{\nu}^{\frac{1}{2}-s}}{1-q_{\nu}^{1-2 s}}-\frac{|2|_{\nu}^{2 s}\left|P\left(\mathrm{x}_{\nu}\right)\right|_{\nu}^{-s}}{1-q_{\nu}^{-2 s}}\right) & \text { if }\left|P\left(\mathrm{x}_{\nu}\right)\right|_{\nu}>|4|_{\nu} \\
\frac{1}{\left(1-q_{\nu}^{-2 s}\right)\left(1-\frac{\left.q_{\nu}^{1-2 s}\right)}{}\right.} & \text { if }\left|P\left(\mathrm{x}_{\nu}\right)\right|_{\nu} \leq|4|_{\nu} .\end{cases}
\end{aligned}
$$

We note that for a standard orbital representative $\mathrm{x}_{\nu}$ of a form $x \in V_{K}$, $\left|P\left(\mathrm{x}_{\nu}\right)\right|_{\nu}^{-1}$ is just the norm of the local discriminant of $K_{x}$ over $K$ at $\nu$.

We are now ready to state

Proposition 1.4. Let $M_{2}(K)$ denote the set of all places of $K$ that lie over 2. Then

$$
\eta_{x, S}^{*}(s)=\prod_{\nu \in M_{2}(K)-S} Z_{\mathrm{x}_{\nu}}\left(s, \Phi_{0, \nu}^{*}\right) \eta_{x, S \cup M_{2}(K)}(s)
$$

where $\mathrm{x}_{\nu}$ is the standard orbital representative of $x$ at $\nu$ and the values of $Z_{\mathrm{x}_{\nu}}\left(s, \Phi_{0, \nu}^{*}\right)$ are given in (1.22) and (1.23).

Propositions 1.3 and 1.4 complete our discussion of the Dirichlet series $\xi_{\mathrm{x}_{s}}(s)$ and $\xi_{\mathrm{x}_{s}}^{*}(s)$ and of their origins.

\section{The functional equation coefficients}

\subsection{The split case}


By (1.11),

$$
\Gamma_{\mathrm{x}_{\nu} \mathrm{y}_{\nu}}=\frac{Z_{\mathrm{y}_{\nu}}\left(s, \Phi_{\nu}^{*}\right)}{Z_{\mathrm{x}_{\nu}}\left(\frac{3}{2}-s, \Phi_{\nu}\right)}
$$

where $\Phi_{\nu}$ is any test function with support in $V_{\mathrm{x}_{\nu}}$.

The notation $\Gamma_{\mathrm{x}_{\nu} \mathrm{y}_{\nu}}$ is rather cumbersome, and we are going to change it in this section. We will index the non-singular orbits in $V_{K_{\nu}}$ as follows: $V_{1}$ will stand for the orbit of forms in $V_{K_{\nu}}^{\prime}$ that split over $K_{\nu} . V_{2}$ will denote the orbit of those forms whose splitting field is a quadratic unramified extension of $K_{\nu}$. If $\nu \Varangle 2$, we will denote by $V_{2(1)}$ and $V_{2(2)}$ the orbits of forms whose splitting fields are the two quadratic ramified extensions of $K_{\nu}$. If $\nu \mid 2$, the number of quadratic ramified extensions of $K_{\nu}$ is greater than two; we will introduce the notations for orbits of forms corresponding to these extensions in the appropriate section.

We will write $\mathrm{x}_{i}$ for the standard orbital representative of the orbit $V_{i}, Z_{i}$ (s, $\left.\Phi_{\nu}\right)$ for $Z_{\mathrm{x}_{i}}\left(s, \Phi_{\nu}\right)$ and $\Gamma_{i j}^{\nu}(s)$ for $\Gamma_{\mathrm{x}_{i} \mathrm{x}_{j}}(s)$. In this section we are going to determine the coefficients $\Gamma_{j 1}^{\nu}(s)$.

For any $K_{\nu}$, the standard orbital representative for the orbit $V_{1}$ is $\mathrm{x}_{1}=$ $(0,1,0) . H_{\mathrm{x}_{1}}^{0}=\left\{\varrho\left(d\left(\tau_{\nu}^{-1}, 1\right) a\left(\tau_{\nu}\right)\right): \tau_{\nu} \in K_{\nu}^{\times}\right\}$, the measure $d_{\mathrm{x}_{1}}^{\prime \prime} h_{\nu}^{\prime \prime}=d_{\nu}^{\times} \tau_{\nu}$, and $d_{\mathrm{x}_{1}}^{\prime}$ $h_{\nu}^{\prime}=d \kappa_{\nu} d^{\times} t_{\nu} d u_{\nu}$. Note that $d(t, 1) n(u) \cdot(0,1,0)=(0, t, t u)$ and that integration with respect to $d \kappa_{\nu}$ simply replaces $\Phi_{\nu}$ by $M_{\nu} \Phi_{\nu}$, where $M_{\nu} \Phi_{\nu}\left(x_{\nu}\right)=$ $\int_{\mathscr{K}_{\nu}} \Phi_{\nu}\left(\kappa_{\nu} \cdot x_{\nu}\right) d \kappa_{\nu}$. Therefore

$$
\begin{aligned}
Z_{1}\left(s, \Phi_{\nu}\right) & =\int_{K_{\nu}^{\times}} \int_{K_{\nu}}\left|t_{\nu}\right|_{\nu}^{2 s}\left(M_{\nu} \Phi_{\nu}\right)\left(0, t_{\nu}, t_{\nu} u_{\nu}\right) d u_{\nu} d^{\times} t_{\nu} \\
& =\int_{K_{\nu}^{\times}} \int_{K_{\nu}}\left|t_{\nu}\right|_{\nu}^{2 s-1}\left(M_{\nu} \Phi_{\nu}\right)\left(0, t_{\nu}, u_{\nu}\right) d u_{\nu} d^{\times} t_{\nu} .
\end{aligned}
$$

Let $I_{3} \Phi_{\nu}\left(t_{\nu}\right)=\int_{K_{\nu}} \Phi_{\nu}\left(0, t_{\nu}, u_{\nu}\right) d u_{\nu}$. Then $I_{3} \Phi_{\nu}$ is a Schwartz-Bruhat function on $K_{\nu}$, and for any $\mathscr{K}_{\nu}$-invariant function $\Phi_{\nu}$

$$
Z_{1}\left(s, \Phi_{\nu}\right)=\zeta_{\nu}\left(2 s-1, I_{3} \Phi_{\nu}\right)
$$

where $\zeta_{\nu}\left(s, \Psi_{\nu}\right)$ is the local zeta function of Tate's thesis [13].

The following lemma is an easy exercise in Fourier inversion:

LEMma 2.1. $\left(I_{3} \Phi_{\nu}\right)^{*}(t)=\left(I_{3} \Phi_{\nu}^{*}\right)(-2 t)$.

Note that if $\Phi_{\nu}$ is $\mathscr{K}_{\nu}$-invariant, so is its Fourier transform. Also, for any $t_{\nu} \in$ $K_{\nu}^{\times}, u_{\nu} \in K_{\nu},\left(0, t_{\nu}, u_{\nu}\right) \in V_{1}$. Hence for any $\Phi_{\nu}$ with support in $V_{j}, j \neq 1, I_{3} \Phi_{\nu}$ 
$=0$. By Lemma $2.1 I_{3} \Phi_{\nu}^{*}=0$. Hence $Z_{1}\left(s, \Phi_{\nu}^{*}\right)=0$. Thus for any $j \neq 1$, $\Gamma_{j 1}^{\nu}(s)=0$.

By (2.1), (2.3) and Lemma 2.1

$$
\Gamma_{11}^{\nu}(s)=\frac{|2|_{\nu}^{2 s-1} \zeta_{\nu}\left(2 s-1,\left(I_{3} \Phi_{\nu}\right)^{*}\right)}{\zeta_{\nu}\left(2-2 s, I_{3} \Phi_{\nu}\right)}
$$

The last quotient is well known (see [13] or [15]). We now have:

Proposition 2.2.

$$
\Gamma_{j 1}^{\nu}(s)=\delta_{j 1}|2|_{\nu}^{2 s-1} \frac{\Gamma_{\nu}(2 s-1)}{\Gamma_{\nu}(2-2 s)}
$$

where $\delta_{j 1}$ is the Kronecker delta and

$$
\begin{aligned}
& \Gamma_{\nu}(s)= \begin{cases}\pi^{-\frac{s}{2}} \Gamma\left(\frac{s}{2}\right) & \text { if } K_{\nu}=\mathbf{R} ; \\
(2 \pi)^{-s} \Gamma(s) & \text { if } K_{\nu}=\mathbf{C} ;\end{cases} \\
& \left(1-q_{\nu}^{-s}\right)^{-1} \text { if } K_{\nu} \text { is non-archimedean. }
\end{aligned}
$$

\subsection{The archimedean completions}

For $K_{\nu}=\mathbf{R}$, the local functional equation coefficients were found by Shintani [12]. We state his result as a propositin below:

Proposition 2.3. For $K_{\nu}=\mathbf{R}$,

$$
\left(\begin{array}{ll}
\Gamma_{11}^{\nu}(s) & \Gamma_{12}^{\nu}(s) \\
\Gamma_{21}^{\nu}(s) & \Gamma_{22}^{\nu}(s)
\end{array}\right)=2^{2 s-1} \pi^{\frac{1}{2}-2 s} \Gamma\left(s-\frac{1}{2}\right) \Gamma(s)\left(\begin{array}{cc}
\sin \pi s & 1 \\
0 & \cos \pi s
\end{array}\right) .
$$

We note that Shintani's $\Gamma_{11}^{\nu}(s)$ agrees with $\Gamma_{11}^{\nu}(s)$ we found in Proposition 2.2. For $K_{\nu}=\mathbf{C}$, the value of $\Gamma_{11}^{\nu}(s)$ can be found in Proposition 2.2.

\section{3. $\mathbf{p}$-adic completions, $\mathbf{p} \neq \mathbf{2}$}

Let $K_{\nu}, \nu \times 2$, be a non-archimedean completion of $K$. Let $\Phi_{i, \nu}$ denote the characteristic function of $\mathscr{K}_{\nu} \cdot \mathrm{x}_{i}$. By [3], Section $4.2, Z_{i}\left(s, \Phi_{i, \nu}\right)=1$. Therefore by $(2.1)$

$$
\Gamma_{i j}^{\nu}(s)=Z_{j}\left(s, \Phi_{i, \nu}^{*}\right) .
$$

Let $\mathbf{F}_{q_{\nu}}$ denote the residue field of $K_{\nu}$. Then for $i=1,2 \mathscr{K}_{\nu} \cdot x_{i}$ can be described as follows: 


$$
\begin{aligned}
\mathscr{K}_{\nu} \cdot \mathrm{x}_{1}=\{x & \equiv t(u+a v)(u+b v) \text { or } \\
x & \left.\equiv t v(u+a v) \bmod \pi_{\nu}: t \in \mathbf{F}_{q_{\nu}}^{\times}, a, b \in \mathbf{F}_{q_{\nu}}, a \neq b\right\},
\end{aligned}
$$

and

(2.7) $\mathscr{K}_{\nu} \cdot \mathrm{x}_{2}=\left\{x \equiv t(u+\alpha v)\left(u+\alpha^{\prime} v\right) \bmod \pi_{\nu}: t \in \mathbf{F}_{q_{\nu}}^{\times}, \alpha \in \mathbf{F}_{q_{\nu}^{2}}-\mathbf{F}_{q_{\nu}}\right\}$

where $\alpha^{\prime}$ denotes the Galois conjugate of $\alpha$ over $\mathbf{F}_{q_{\nu}}$.

Clearly, $\Phi_{i, \nu}^{*}(x), i=1,2$, has support in $\pi_{\nu}^{-1} V_{O_{\nu}}$, depends only on $x$ modulo $O_{\nu}$ and is $\mathscr{K}_{\nu}$-invariant. This implies that $\Phi_{i, \nu}^{*}(x)$ takes on four distinct values on the following sets: $V_{O_{\nu}}, \pi_{\nu}^{-1}\left(\mathscr{K}_{\nu} \cdot \mathrm{x}_{i}\right), i=1,2$, and $\pi_{\nu}^{-1}\left(\mathscr{K}_{\nu} \cdot(0,0,1)\right)$.

Let $x_{a, b}=(u+a v)(u+b v)$ and $x_{a, \infty}=v(u+a v)$. Then

$$
\Phi_{1, \nu}^{*}(y)=q_{\nu}^{-3} \sum_{a \neq b \in \mathbf{F}_{q \nu} \cup\{\infty\}} \sum_{t \in \mathbf{F}_{q \nu}^{\times}}\left\langle t\left[x_{a, b}, y\right]\right\rangle_{\nu} .
$$

The inner sum in (2.8) is either $q_{\nu}-1$ or -1 depending on whether $\left[x_{a, b}, y\right]$ is in $O_{\nu}$ or not. Hence

$$
\Phi_{1, \nu}^{*}(x)= \begin{cases}\frac{1-q_{\nu}^{-2}}{2} & \text { if } x \in V_{O_{\nu}} \\ -q_{\nu}^{-2} & \text { if } x \in \pi_{\nu}^{-1}\left(\mathscr{K}_{\nu} \cdot \mathrm{x}_{1}\right) ; \\ 0 & \text { if } x \in \pi_{\nu}^{-1}\left(\mathscr{K}_{\nu} \cdot \mathrm{x}_{2}\right) ; \\ \frac{q_{\nu}^{-1}\left(1-q_{\nu}^{-1}\right)}{2} & \text { if } x \in \pi_{\nu}^{-1}\left(\mathcal{K}_{\nu} \cdot(0,0,1)\right) .\end{cases}
$$

Another way to write $\Phi_{1, \nu}$ is the following:

$$
\begin{aligned}
\Phi_{1, \nu}^{*}= & \frac{q_{\nu}^{-1}\left(1-q_{\nu}^{-1}\right)}{2}\left(\pi_{\nu}^{-1} \cdot \Phi_{0, \nu}\right)+\frac{\left(1-q_{\nu}^{-1}\right)}{2} \Phi_{0, \nu} \\
& -\frac{q_{\nu}^{-1}\left(1+q_{\nu}^{-1}\right)}{2}\left(\pi_{\nu}^{-1} \cdot \Phi_{1, \nu}\right)-\frac{q_{\nu}^{-1}\left(1-q_{\nu}^{-1}\right)}{2}\left(\pi_{\nu}^{-1} \cdot \Phi_{2, \nu}\right)
\end{aligned}
$$

where $(t \cdot \Phi)(x)=\Phi\left(t^{-1} x\right)$.

A similar argument shows that

$$
\Phi_{2, \nu}^{*}(x)= \begin{cases}\frac{\left(1-q_{\nu}^{-1}\right)^{2}}{2} & \text { if } x \in V_{O_{\nu}} ; \\ 0 & \text { if } x \in \pi_{\nu}^{-1}\left(\mathscr{K}_{\nu} \cdot \mathrm{x}_{1}\right) ; \\ q_{\nu}^{-2} & \text { if } x \in \pi_{\nu}^{-1}\left(\mathscr{K}_{\nu} \cdot \mathrm{x}_{2}\right) ; \\ -\frac{q_{\nu}^{-1}\left(1-q_{\nu}^{-1}\right)}{2} & \text { if } x \in \pi_{\nu}^{-1}\left(\mathscr{K}_{\nu} \cdot(0,0,1)\right) .\end{cases}
$$


Therefore

$$
\begin{aligned}
\Phi_{2, \nu}^{*}= & -\frac{q_{\nu}^{-1}\left(1-q_{\nu}^{-1}\right)}{2}\left(\pi_{\nu}^{-1} \cdot \Phi_{0, \nu}\right)+\frac{\left(1-q_{\nu}^{-1}\right)}{2} \Phi_{0, \nu} \\
& +\frac{q_{\nu}^{-1}\left(1-q_{\nu}^{-1}\right)}{2}\left(\pi_{\nu}^{-1} \cdot \Phi_{1, \nu}\right)+\frac{q_{\nu}^{-1}\left(1+q_{\nu}^{-1}\right)}{2}\left(\pi_{\nu}^{-1} \cdot \Phi_{2, \nu}\right)
\end{aligned}
$$

Next we compute $\Phi_{2(i), \nu}^{*}(s), i=1,2$. We may assume that the first quadratic ramified extensions of $K_{\nu}$ is the splitting field of the form $u^{2}+\pi_{\nu} v^{2}$ and the second the splitting field of $u^{2}+\alpha_{\nu} \pi_{\nu} v^{2}$ where $\alpha_{\nu} \in O_{\nu}^{\times}$is a non-square. Let $\Psi_{2(1), \nu}(x)$ be the characteristic function of the set of Eisenstein polynomials $\left\{x \equiv t\left(u^{2}+\right.\right.$ $\left.a^{2} \pi_{\nu} v^{2}\right) \bmod \left(\pi_{\nu} \times \pi_{\nu} \times \pi_{\nu}^{2}\right): t \in \mathbf{F}_{q_{\nu}}^{\times}, a \in \mathbf{F}_{q_{\nu}}^{\times}$. Then $\Phi_{2(1), \nu}=\left(q_{\nu}+1\right) M_{\nu} \Psi_{2(1), \nu}$.

$\Psi_{2(1), \nu}^{*}$ is easy to compute. Its support lies in $\pi_{\nu}^{-2} O_{\nu} \times \pi_{\nu}^{-1} O_{\nu} \times \pi_{\nu}^{-1} O_{\nu}$. Moreover, for any $y \in \pi_{\nu}^{-2} O_{\nu} \times \pi_{\nu}^{-1} O_{\nu} \times \pi_{\nu}^{-1} O_{\nu}$

$$
\Psi_{2(1), \nu}^{*}(y)=q_{\nu}^{-4}\left(\left(q_{\nu}-1\right) \#\left\{x_{a}:\left[y, x_{a}\right] \in O_{\nu}\right\}-\#\left\{x_{a}:\left[y, x_{a}\right] \notin O_{\nu}\right\}\right)
$$

where $x_{a}$ denotes the form $u^{2}+a^{2} \pi_{\nu} v^{2}, a \in \mathbf{F}_{q_{\nu}}$. An easy computation now shows that for $y \in \pi_{\nu}^{-2} O_{\nu} \times \pi_{\nu}^{-1} O_{\nu} \times \pi_{\nu}^{-1} O_{\nu}$

$$
\Psi_{2(1), \nu}^{*}(y)=\left\{\begin{array}{cc}
q_{\nu}^{-4} \frac{\left(q_{\nu}-1\right)^{2}}{2} & \text { if } y \in \pi_{\nu}^{-1} O_{\nu} \times \pi_{\nu}^{-1} O_{\nu} \times O_{\nu} \\
q_{\nu}^{-4} \frac{\left(q_{\nu}+1\right)}{2} & \text { if } y \in \pi_{\nu}^{-2} O_{\nu}^{\times} \times \pi_{\nu}^{-1} O_{\nu} \times \pi_{\nu}^{-1} O_{\nu}^{\times} \\
& \pi_{\nu}^{2} y \equiv \tau\left(u^{2}-b^{2} \pi_{\nu} v^{2}\right) \bmod \pi_{\nu} \times \pi_{\nu} \times \pi_{\nu}^{2} \\
-q_{\nu}^{-4} \frac{\left(q_{\nu}-1\right)}{2} & \text { otherwise. }
\end{array}\right.
$$

Let $F_{1, \nu}$ and $F_{2, \nu}$ be the characteristic functions of $O_{\nu} \times O_{\nu} \times \pi_{\nu} O_{\nu}$ and $O_{\nu} \times$ $\pi_{\nu} O_{\nu} \times \pi_{\nu} O_{\nu}$ respectively. Then

$$
\Psi_{2(1), \nu}^{*}=-q_{\nu}^{-4} \frac{\left(q_{\nu}-1\right)}{2} \pi_{\nu}^{-2} \cdot F_{2, \nu}+q_{\nu}^{-3} \frac{\left(q_{\nu}-1\right)}{2} \pi_{\nu}^{-1} \cdot F_{1, \nu}+q_{\nu}^{-3} \pi_{\nu}^{-2} \cdot \Psi_{2(j), \nu}
$$

where $j=1$ if $q_{\nu} \equiv 1 \bmod 4$ (i.e. -1 is a square in $\mathbf{F}_{q_{\nu}}$ ) and $j=2$ otherwise.

LEMma 2.4.

$$
\begin{aligned}
& M_{\nu} F_{2, \nu}=\frac{1}{q_{\nu}+1}\left(\Phi_{0, \nu}+q_{\nu}\left(\pi_{\nu} \cdot \Phi_{0, \nu}\right)-\Phi_{1, \nu}-\Phi_{2, \nu}\right) ; \\
& M_{\nu} F_{1, \nu}=\frac{1}{q_{\nu}+1}\left(\Phi_{0, \nu}+q_{\nu}\left(\pi_{\nu} \cdot \Phi_{0, \nu}\right)+\Phi_{1, \nu}-\Phi_{2, \nu}\right) .
\end{aligned}
$$


Proof. $F_{2, \nu}=\pi_{\nu} \cdot \Phi_{0, \nu}+F_{3, \nu}$ where $F_{3, \nu}$ is the characteristic function of the set $S_{3, \nu}=\left\{x \equiv(t, 0,0) \bmod \pi_{\nu}: t \in \mathbf{F}_{q_{\nu}}^{\times}\right.$. The stabilizer of this set has index $q_{\nu}+1$ in $\mathscr{K}_{\nu}$, any two distinct $\kappa_{\nu} \cdot S_{3, \nu}, \kappa_{\nu} \in \mathscr{K}_{\nu}$ are disjoint, and the image of $S_{3, \nu}$ under the action of $\mathscr{K}_{\nu}$ consists of those forms in $O_{\nu}^{3}-\left(\pi_{\nu} O_{\nu}\right)^{3}$ that are singular modulo $\pi_{\nu}$. Therefore

$$
M_{\nu} F_{3, \nu}=\frac{1}{q_{\nu}+1}\left(\Phi_{0, \nu}-\left(\pi_{\nu} \cdot \Phi_{0, \nu}\right)-\Phi_{1, \nu}-\Phi_{2, \nu}\right)
$$

and $M_{\nu} F_{2, \nu}=M_{\nu} F_{3, \nu}+\pi_{\nu} \cdot \Phi_{0, \nu}$ is as in the statement of the lemma.

$F_{1, \nu}=F_{2, \nu}+F_{4, \nu}$ where $F_{4, \nu}$ is the characteristic function of the set

$$
S_{4, \nu}=\left\{x \equiv\left(t_{1}, t_{2}, 0\right) \bmod \pi_{\nu}: t_{2} \in O_{\nu}^{\times}\right\} .
$$

Alternatively,

$$
S_{4, \nu}=\left\{\kappa_{\nu} \cdot(0,1,0): \kappa_{\nu}=\left(t,\left(\begin{array}{ll}
a & b \\
c & d
\end{array}\right)\right) \in \mathscr{K}_{\nu}, c \equiv 0 \bmod \pi_{\nu} \text { ord } \equiv 0 \bmod \pi_{\nu}\right\}
$$

Hence $M_{\nu} F_{4, \nu}=\frac{2}{q_{\nu}+1} \Phi_{1, \nu}$. Adding this to the value of $M_{\nu} F_{2, \nu}$, we obtain $M_{\nu} F_{1, \nu}$.

From (2.14) and Lemma 2.4

$$
\begin{aligned}
\Phi_{2(1), \nu}^{*} & =q_{\nu}^{-4} \frac{\left(q_{\nu}-1\right)}{2}\left(-\pi_{\nu}^{-2} \cdot \Phi_{0, \nu}+q_{\nu}^{2} \Phi_{0, \nu}\right) \\
& +q_{\nu}^{-4} \frac{\left(q_{\nu}-1\right)}{2}\left(\pi_{\nu}^{-2} \cdot \Phi_{1, \nu}+q_{\nu} \pi_{\nu}^{-1} \cdot \Phi_{1, \nu}\right) \\
& +q_{\nu}^{-4} \frac{\left(q_{\nu}-1\right)}{2}\left(\pi_{\nu}^{-2} \cdot \Phi_{2, \nu}-q_{\nu} \pi_{\nu}^{-1} \cdot \Phi_{2, \nu}\right)+q_{\nu}^{-3} \pi_{\nu}^{-2} \cdot \Phi_{2(j), \nu}
\end{aligned}
$$

By (1.7) $Z_{i}\left(s, t_{\nu} \cdot \Phi_{\nu}\right)=\left|\chi\left(t_{\nu}\right)\right|_{\nu}^{s} Z_{i}\left(s, \Phi_{\nu}\right)$. Therefore it is now a simple matter to compute $\Gamma_{i j}^{\nu}(s)$ from (2.10), (2.12) and (2.15):

Proposition 2.5. If $\nu \nmid 2$, the local functional equation coefficients $\Gamma_{i j}^{\nu}(s)$, $i, j=1,2,2(1), 2(2)$, are given in the following table:

$$
\begin{aligned}
& \frac{1-q_{\nu}^{2 s-2}}{1-q_{\nu}^{i-2 s}} \frac{1-q_{\nu}^{-2}}{\left(1-q_{\nu}^{-2 s}\right)\left(1-q_{\nu}^{1-2 s}\right)} \quad \frac{\left(1-q_{\nu}^{-1}\right)\left(1+q_{\nu}^{2 s-1}\right)}{2\left(1-q_{\nu}^{-2 s}\right)\left(1-q_{\nu}^{1-2 s}\right)} \quad \frac{\left(1-q_{\nu}^{-1}\right)\left(1+q_{\nu}^{2 s-1}\right)}{2\left(1-q_{\nu}^{-2 s}\right)\left(1-q_{\nu}^{1-2 s}\right)} \\
& 0 \quad \frac{q_{\nu}^{2 s-2}\left(1-q_{\nu}^{1-2 s}\right)}{1-q_{\nu}^{-2 s}} \quad \frac{-q_{\nu}^{2 s-1}\left(1-q_{\nu}^{-1}\right)}{2\left(1-q_{\nu}^{-2 s}\right)} \quad \frac{-q_{\nu}^{2 s-1}\left(1-q_{\nu}^{-1}\right)}{2\left(1-q_{\nu}^{-2 s}\right)}
\end{aligned}
$$


$0-\frac{\left(1-q_{\nu}^{-2}\right) q_{\nu}^{2 s-2}}{1-q_{\nu}^{-2 s}} \frac{\left(1+\left(\frac{-1}{q_{\nu}}\right) q_{\nu}\right) q_{\nu}^{4 s-4}\left(1-\left(\frac{-1}{q_{\nu}}\right) q_{\nu}^{1-2 s}\right)}{2\left(1-q_{\nu}^{-2 s}\right)} \frac{\left(1-\left(\frac{-1}{q_{\nu}}\right) q_{\nu}\right) q_{\nu}^{4 s-4}\left(1+\left(\frac{-1}{q_{\nu}}\right) q_{\nu}^{1-2 s}\right)}{2\left(1-q_{\nu}^{-2 s}\right)}$
$0 \quad-\frac{\left(1-q_{\nu}^{-2}\right) q_{\nu}^{2 s-2}}{1-q_{\nu}^{-2 s}} \frac{\left(1-\left(\frac{-1}{q_{\nu}}\right) q_{\nu}\right) q_{\nu}^{4 s-4}\left(1+\left(\frac{-1}{q_{\nu}}\right) q_{\nu}^{1-2 s}\right)}{2\left(1-q_{\nu}^{-2 s}\right)} \frac{\left(1+\left(\frac{-1}{q_{\nu}}\right) q_{\nu}\right) q_{\nu}^{4 s-4}\left(1-\left(\frac{-1}{q_{\nu}}\right) q_{\nu}^{1-2 s}\right)}{2\left(1-q_{\nu}^{-2 s}\right)}$ where $\left(\frac{-1}{q_{\nu}}\right)$ is the Jacobi symbol.

\subsection{2-adic completions}

Let $K_{\nu}, \nu \mid 2$, be a 2 -adic completion of $K$. Let $m_{\nu}$ denote the integer such that $|2|_{\nu}=q_{\nu}^{-m_{\nu}}$. The quadratic ramified extensions of $K_{\nu}$ have discriminants of norm $q_{\nu}^{2 i}, i=1, \ldots, m_{\nu}$ and $q_{\nu}^{2 m_{\nu}+1}$. We will denote by $V_{2^{i}(k)}$ the orbit of forms whose splitting field is the $k^{t h}$ field in the list of quadratic ramified extensions of $K_{\nu}$ with the discriminant of norm $q_{\nu}^{i}$. A standard orbital representative of $V_{2^{i}(k)}$ will be denoted by $\mathrm{x}_{2^{i}(k)}$. When there is no need to distinguish between distinct ramified extensions of $K_{\nu}$ of discriminant $q_{\nu}^{i}$, we will simply write $V_{2^{i}}$ and $\mathrm{x}_{2^{i}}$ for $V_{2^{i}(k)}$ and $\mathrm{X}_{2^{i}(k)}$.

Denote by $\Phi_{0, \nu}^{j}$ the characteristic function of $\left\{x \in V_{O_{\nu}}:|P(x)|_{\nu} \leq q_{\nu}^{-j}\right\}$. The possible values of $|P(x)|_{\nu}$ are $q_{\nu}^{-2 j}, j=0,1, \ldots, m_{\nu}$, and $q_{\nu}^{-k}, k \geq 2 m_{\nu}+1$. Consequently, we need only distinguish between $\Phi_{0, \nu}^{2 j}, j=0, \ldots, m_{\nu}$, and $\Phi_{0, \nu}^{k}, k \geq 2 m_{\nu}$ +1 . The functions $\Phi_{0, \nu}^{2 j}, j=0, \ldots, m_{\nu}$, afford a rather simple description: they are the characteristic functions of the sets $O_{\nu} \times \pi_{\nu}^{j} O_{\nu} \times O_{\nu}$. In particular, $\Phi_{0, \nu}^{2 m_{\nu}}$ is the characteristic function of $O_{\nu} \times 2 O_{\nu} \times O_{\nu}$.

$$
Z_{2^{i}}\left(s, \Phi_{0, \nu}^{j}\right)=\int_{H_{K_{\nu}}^{i j} /\left(H_{\mathrm{x}_{2} i^{\prime} K_{\nu}}^{0}\right.}|\chi(h)|_{\nu}^{s} \Phi_{0, \nu}\left(h \cdot \mathrm{x}_{2^{i}}\right) d_{\mathrm{x}_{2_{i}}}^{\prime} h \text { where } H_{K_{\nu}}^{i-j}=\left\{h \in H_{K_{\nu}}:\right.
$$
$\left.|\chi(h)|_{\nu}^{2} \leq q_{\nu}^{i-j}\right\}$. The value of $Z_{2^{i}}\left(s, \Phi_{0, \nu}\right)$ is given in Proposition 1.3. It is a simple exercise now to obtain:

$$
\begin{aligned}
& \text { Lemma 2.6. For an integer } j \leq 0 \text { let } \tilde{j}=\left\{\begin{array}{ll}
\frac{j}{2} & \text { if } j \text { even } \\
\frac{j+1}{2} & \text { if } j \text { odd }
\end{array}\right. \text {. Then } \\
& \qquad Z_{1}\left(s, \Phi_{0, \nu}^{j}\right)=\frac{q_{\nu}^{\tilde{j}(1-2 s)}}{1-q_{\nu}^{1-2 s}}, \\
& \qquad Z_{2}\left(s, \Phi_{0, \nu}^{j}\right)=\frac{1}{q_{\nu}-1}\left(\frac{\left(q_{\nu}+1\right) q_{\nu}^{\tilde{j}(1-2 s)}}{1-q_{\nu}^{1-2 s}}-\frac{2 q_{\nu}^{-2 \tilde{j}}}{1-q_{\nu}^{-2 s}}\right),
\end{aligned}
$$


and if $j>i$,

$$
Z_{2^{i}}\left(s, \Phi_{0, \nu}^{j}\right)=\frac{1}{q_{\nu}-1}\left(\frac{q_{\nu} q_{\nu}^{(\widetilde{j-i})(1-2 s)}}{1-q_{\nu}^{1-2 s}}-\frac{q_{\nu}^{-2(\widetilde{j-i}) s}}{1-q_{\nu}^{-2 s}}\right) .
$$

If $j \leq i$,

$$
Z_{2^{i}}\left(s, \Phi_{0, \nu}^{j}\right)=\frac{1}{\left(1-q_{\nu}^{1-2 s}\right)\left(1-q_{\nu}^{-2 s}\right)} .
$$

Let $\Phi_{i, \nu}, i=1,2$, be as in the previous section. Then as before $\Gamma_{i j}^{\nu}(s)=$ $Z_{j}\left(s, \Phi_{i, \nu}^{*}\right)$.

$\Phi_{1, \nu}$ is the characteristic function of the set $\mathscr{K}_{\nu} \cdot \mathrm{x}_{1}$ whose explicit description is given by (2.5). Clearly $\Phi_{1, \nu}^{*}(y)$ has support in $\pi_{\nu}^{-1}\left(O_{\nu} \times 2 O_{\nu} \times O_{\nu}\right)$, depends only on $y$ modulo $O_{\nu} \times 2 O_{\nu} \times O_{\nu}$ and is $\mathscr{K}_{\nu}$-invariant. Therefore we only need to compute $\Phi_{1, \nu}^{*}(y)$ for four distinct values of $y: y=(0,0,0), y=\pi_{\nu}^{-1}(1,0,1), y=$ $\pi_{\nu}^{-1}(1,0,0)$ and $y=\pi_{\nu}^{-1}(0,2,0)$. The computation is carried out as in (2.8): one need only find the number of $x_{a, b}$ such that $\left[y, x_{a, b}\right] \in O_{\nu}$. The result is the following:

$$
\Phi_{1, \nu}^{*}(y)= \begin{cases}\frac{1-q_{\nu}^{-2}}{2} & \text { if } y \in O_{\nu} \times 2 O_{\nu} \times O_{\nu} ; \\ \frac{-q_{\nu}^{-2}}{2} & \text { if } y \equiv \kappa_{\nu} \cdot \pi_{\nu}^{-1}(1,0,1) \bmod O_{\nu} \times 2 O_{\nu} \times O_{\nu}, \kappa_{\nu} \in \mathscr{K}_{\nu} ; \\ \frac{q_{\nu}^{-1}\left(1-q_{\nu}^{-1}\right)}{2} & \text { if } y \equiv \kappa_{\nu} \cdot \pi_{\nu}^{-1}(1,0,0) \bmod O_{\nu} \times 2 O_{\nu} \times O_{\nu}, \kappa_{\nu} \in \mathscr{K}_{\nu} ; \\ \frac{-q_{\nu}^{-1}\left(1+q_{\nu}^{-1}\right)}{2} & \text { if } y \in \kappa_{\nu} \cdot \pi_{\nu}^{-1}(0,2,0) \bmod O_{\nu} \times 2 O_{\nu} \times O_{\nu}, \kappa_{\nu} \in \mathscr{K}_{\nu} .\end{cases}
$$

The last three sets in (2.16) look rather complicated. In fact, they have better descriptions. The first consists of $x \in \pi_{\nu}^{-1}\left(O_{\nu} \times 2 O_{\nu} \times O_{\nu}\right)-O_{\nu}^{3}$ such that $|P(x)|_{\nu}=q_{\nu}^{2-2 m_{\nu}}$, the second consists of $x \in \pi_{\nu}^{-1}\left(O_{\nu} \times 2 O_{\nu} \times O_{\nu}\right)-O_{\nu}^{3}$ such that $|P(x)|_{\nu} \leq q_{\nu}^{1-2 m_{\nu}}$, and the third is the set of $x \in O_{\nu}^{3}$ such that $|P(x)|_{\nu}=q_{\nu}^{2-2 m_{\nu}}$.

Let $F_{i, \nu}, i=1,2,3$, be the characteristic functions of these three sets. Then

$$
\Phi_{1, \nu}^{*}=-\frac{q_{\nu}^{-2}}{2} \pi_{\nu}^{-1} \cdot \Phi_{0, \nu}^{2 m_{\nu}}+\frac{1}{2} \Phi_{0, \nu}^{2 m_{\nu}}+\frac{q_{\nu}^{-1}}{2} F_{2, \nu}-\frac{q_{\nu}^{-1}}{2} F_{3, \nu} .
$$

Now $F_{2, \nu}=\pi_{\nu}^{-1} \cdot \Phi_{0, \nu}^{2 m_{\nu}+1}-\Phi_{0, \nu}^{2 m_{\nu}-1}$ and $F_{3, \nu}=\Phi_{0, \nu}^{2 m_{\nu}-2}-\Phi_{0, \nu}^{2 m_{\nu}-1}$. Hence

$$
\Phi_{1, \nu}^{*}=-\frac{q_{\nu}^{-2}}{2} \pi_{\nu}^{-1} \cdot \Phi_{0, \nu}^{2 m_{\nu}}+\frac{1}{2} \Phi_{0, \nu}^{2 m_{\nu}}+\frac{q_{\nu}^{-1}}{2} \pi_{\nu}^{-1} \cdot \Phi_{0, \nu}^{2 m_{\nu}+1}-\frac{q_{\nu}^{-1}}{2} \Phi_{0, \nu}^{2 m_{\nu}-2} .
$$

Similarly, 
$\Phi_{2, \nu}^{*}(y)= \begin{cases}\frac{\left(1-q_{\nu}^{-1}\right)^{2}}{2} & \text { if } y \in O_{\nu} \times 2 O_{\nu} \times O_{\nu} ; \\ \frac{q_{\nu}^{-2}}{2} & \text { if } y \equiv \kappa_{\nu} \cdot \pi_{\nu}^{-1}(1,0,1) \bmod O_{\nu} \times 2 O_{\nu} \times O_{\nu}, \kappa_{\nu} \in \mathscr{K}_{\nu} ; \\ \frac{-q_{\nu}^{-1}\left(1-q_{\nu}^{-1}\right)}{2} & \text { if } y \equiv \kappa_{\nu} \cdot \pi_{\nu}^{-1}(1,0,0) \bmod O_{\nu} \times 2 O_{\nu} \times O_{\nu}, \kappa_{\nu} \in \mathscr{K}_{\nu} ; \\ \frac{-q_{\nu}^{-1}\left(1-q_{\nu}^{-1}\right)}{2} & \text { if } y \in \kappa_{\nu} \cdot \pi_{\nu}^{-1}(0,2,0) \bmod O_{\nu} \times 2 O_{\nu} \times O_{\nu}, \kappa_{\nu} \in \mathscr{K}_{\nu},\end{cases}$ or alternatively

$$
\begin{aligned}
\Phi_{2, \nu}^{*} & =\frac{q_{\nu}^{-2}}{2} \pi_{\nu}^{-1} \cdot \Phi_{0, \nu}^{2 m_{\nu}}+\frac{1-2 q_{\nu}^{-1}}{2} \Phi_{0, \nu}^{2 m_{\nu}}-\frac{q_{\nu}^{-1}}{2} F_{2, \nu}-\frac{q_{\nu}^{-1}}{2} F_{3, \nu} \\
& =\frac{q_{\nu}^{-2}}{2} \pi_{\nu}^{-1} \cdot \Phi_{0, \nu}^{2 m_{\nu}}+\frac{1}{2} \Phi_{0, \nu}^{2 m_{\nu}}-\frac{q_{\nu}^{-1}}{2} \pi_{\nu}^{-1} \cdot \Phi_{0, \nu}^{2 m_{\nu}+1}-\frac{q_{\nu}^{-1}}{2} \Phi_{0, \nu}^{2 m_{\nu}-2}
\end{aligned}
$$

The coefficients $\Gamma_{i j}^{\nu}(s), i=1,2$, can now be computed with the help of Lemma 2 .

PROPOSITION 2.7 .

$$
\begin{gathered}
\Gamma_{12}^{\nu}(s)=\frac{q_{\nu}+1}{q_{\nu}-1}\left(\frac{|2|_{\nu}^{2 s-1}\left(1-q_{\nu}^{2 s-2}\right)}{1-q_{\nu}^{1-2 s}}+\frac{q_{\nu}^{2 s-2}|2|_{\nu}^{2 s}\left(1-q_{\nu}^{1-2 s}\right)}{1-q_{\nu}^{-2 s}}\right), \\
\Gamma_{22}^{\nu}(s)=\frac{q_{\nu}^{2 s-2}|2|_{\nu}^{2 s}\left(1-q_{\nu}^{1-2 s}\right)}{1-q_{\nu}^{-2 s}} .
\end{gathered}
$$

For $i=1, \ldots, m_{\nu}-1$,

$$
\Gamma_{12^{2 i}}^{\nu}(s)=\frac{q_{\nu}}{q_{\nu}-1} \frac{q_{\nu}^{i(2 s-1)}|2|_{\nu}^{2 s-1}\left(1-q_{\nu}^{2 s-2}\right)}{1-q_{\nu}^{1-2 s}}+\frac{q_{\nu}+1}{2\left(q_{\nu}-1\right)} \frac{q_{\nu}^{2(i+1) s-2}|2|_{\nu}^{2 s}\left(1-q_{\nu}^{1-2 s}\right)}{1-q_{\nu}^{-2 s}},
$$

and

$$
\begin{gathered}
\Gamma_{22^{2 i}}^{\nu}(s)=\frac{q_{\nu}^{2(i+1) s-2}|2|_{\nu}^{2 s}\left(1-q_{\nu}^{1-2 s}\right)}{2\left(1-q_{\nu}^{-2 s}\right)} . \\
\Gamma_{12^{2 m_{\nu}}}^{\nu}=\frac{2-q_{\nu}^{2 s-2}-q_{\nu}^{-2 s}}{2\left(1-q_{\nu}^{1-2 s}\right)\left(1-q_{\nu}^{-2 s}\right)}, \\
\Gamma_{22^{2 m_{\nu}}}^{\nu}=\frac{q_{\nu}^{2 s-2}\left(1-q_{\nu}^{1-2 s}\right)}{2\left(1-q_{\nu}^{-2 s}\right)}, \\
\Gamma_{12^{2 m_{\nu}+1}}^{\nu}=\frac{\left(q_{\nu}-1\right) q_{\nu}^{2 s-2}\left(1+q_{\nu}^{1-2 s}\right)}{2\left(1-q_{\nu}^{1-2 s}\right)\left(1-q_{\nu}^{-2 s}\right)},
\end{gathered}
$$


and

$$
\Gamma_{22^{2 m_{\nu}+1}}^{\nu}=-\frac{\left(q_{\nu}-1\right) q_{\nu}^{2 s-2}}{2\left(1-q_{\nu}^{-2 s}\right)} .
$$

It remains to compute the local functional equation coefficients for the orbits corresponding to the ramified extensions of $K_{\nu}$. Because of difficulty of dealing with individual ramified extensions we are going to combine all quadratic ramified extensions of $K_{\nu}$ with discriminant of the same norm together. Consequently, let $\Phi_{2^{j}, \nu}$ be the characteristic function of $\cup_{k} \mathscr{K}_{\nu} \cdot \mathrm{x}_{2^{j}(k)}$. Then $\Gamma_{i i^{2}}^{\nu}(s)=Z_{i}\left(s, \Phi_{2^{j}, \nu}^{*}\right)$.

Let $\Psi_{j, \nu}$ denote the characteristic function of the set $O_{\nu}^{\times} \times \pi_{\nu}^{j} O_{\nu} \times \pi_{\nu} O_{\nu}^{\times}$. Then, for $j=1, \ldots, m_{\nu}, \Phi_{2^{2 j, \nu}}=\left(q_{\nu}+1\right) M_{\nu}\left(\Psi_{j, \nu}-\Psi_{j+1, \nu}\right)$ and $\Phi_{2^{2 m_{\nu}+1, \nu}}=\left(q_{\nu}+1\right) M_{\nu}$. $\Psi_{m_{\nu}+1, \nu}$. The Fourier transform of $\Psi_{j, \nu}$ is rather easy to find. The calculation is analogous to the calculation of $\Psi_{2(1), \nu}^{*}$ in Section 2.3. $\operatorname{Supp}\left(\Psi_{j, \nu}\right)^{*} \subset \pi_{\nu}^{-2} O_{\nu} \times$ $\pi_{\nu}^{m_{\nu}-j} O_{\nu} \times \pi_{\nu}^{-1} O_{\nu}$, and for $y \in \pi_{\nu}^{-2} O_{\nu} \times \pi_{\nu}^{m_{\nu}-j} O_{\nu} \times \pi_{\nu}^{-1} O_{\nu}$ one obtains:

$$
\Psi_{j, \nu}^{*}(y)= \begin{cases}q_{\nu}^{-(j+1)}\left(1-q_{\nu}^{-1}\right)^{2} & \text { if } y \in \pi_{\nu}^{-1} O_{\nu} \times \pi_{\nu}^{m_{\nu}-j} O_{\nu} \times O_{\nu} \\ q_{\nu}^{-(j+3)} & \text { if } y \in \pi_{\nu}^{-2} O_{\nu}^{\times} \times \pi_{\nu}^{m_{\nu}-j} O_{\nu} \times \pi_{\nu}^{-1} O_{\nu}^{\times} \\ -q_{\nu}^{-(j+2)}\left(1-q_{\nu}^{-1}\right)^{2} & \text { otherwise. }\end{cases}
$$

Let $\mathscr{F}_{k, \nu}$ be the characteristic function of $O_{\nu} \times \pi_{\nu}^{k} O_{\nu} \times \pi_{\nu} O_{\nu}$. Then

$$
\Psi_{j, \nu}^{*}=-\frac{1-q_{\nu}^{-1}}{q_{\nu}^{j+2}} \pi_{\nu}^{-2} \cdot \mathscr{F}_{m_{\nu}-j+2, \nu}+\frac{1-q_{\nu}^{-1}}{q_{\nu}^{j+1}} \pi_{\nu}^{-1} \cdot \mathscr{F}_{m_{\nu}-j+1, \nu}+\frac{1}{q_{\nu}^{j+2}} \pi_{\nu}^{-2} \cdot \Psi_{m_{\nu}-j 2, \nu}
$$

LEMMA 2.8. For $1 \leq k \leq m_{\nu}$,

$$
\begin{aligned}
M_{\nu} \mathscr{F}_{k, \nu} & =\frac{1}{q_{\nu}+1} \Phi_{0, \nu}^{2 k}+\frac{q_{\nu}}{q_{\nu}+1} \pi_{\nu} \cdot \Phi_{0, \nu}^{2 k-2}, \\
M_{\nu} \mathscr{F}_{m_{\nu}+1, \nu} & =\frac{1}{q_{\nu}+1} \Phi_{0, \nu}^{2 m_{\nu}+1}+\frac{q_{\nu}}{q_{\nu}+1} \pi_{\nu} \cdot \Phi_{0, \nu}^{2 m_{\nu}},
\end{aligned}
$$

and

$$
M_{\nu} \mathscr{F}_{0, \nu}=\frac{1}{q_{\nu}+1} \Phi_{0, \nu}^{2}+\frac{q_{\nu}}{q_{\nu}+1} \pi_{\nu} \cdot \Phi_{0, \nu}+\frac{2}{q_{\nu}+1} \Phi_{1, \nu}
$$

Proof. For $1 \leq k \leq m_{\nu}$,

$$
O_{\nu} \times \pi_{\nu}^{k} O_{\nu} \times \pi_{\nu} O_{\nu}=O_{\nu}^{\times} \times \pi_{\nu}^{k} O_{\nu} \times \pi_{\nu} O_{\nu} \cup \pi_{\nu} O_{\nu} \times \pi_{\nu}^{k} O_{\nu} \times \pi_{\nu} O_{\nu}
$$

The second of the two sets in $(2.21)$ is $\mathscr{K}_{\nu}$-stable; its characteristic function is 
$\pi_{\nu} \cdot \Phi_{0, \nu}^{2 k-2}$. The $\mathscr{K}_{\nu}$-image of the first set in (2.21) is the set of all forms in $O_{\nu} \times$ $\pi_{\nu}^{k} O_{\nu} \times O_{\nu}$ that are not congruent to $0 \bmod \pi_{\nu}$, i.e. it is $\left(O_{\nu} \times \pi_{\nu}^{k} O_{\nu} \times \pi_{\nu} O_{\nu}\right)$ $\left(\pi_{\nu} \cdot O_{\nu} \times \pi_{\nu}^{k} O_{\nu} \times \pi_{\nu} O_{\nu}\right)$. Its stabilizer has index $q_{\nu}+1$ in $\mathscr{K}_{\nu}$. Therefore its characteristic function, normalized with respect to $\mathscr{K}_{\nu}$ is $\frac{1}{q_{\nu}+1}\left(\Phi_{0, \nu}^{2 k}-\pi_{\nu} \cdot \Phi_{0, \nu}^{2 k-2}\right)$. The expression for $M_{\nu} \mathscr{F}_{k, \nu}, k=1, \ldots, m_{\nu}$, is just the sum of the above two expressions.

The argument for $k=m_{\nu}+1$ is similar. As for $k=0, \mathscr{F}_{0, \nu}$ is the characteristic function of

$$
O_{\nu} \times O_{\nu} \times \pi_{\nu} O_{\nu}=O_{\nu} \times \pi_{\nu} O_{\nu} \times \pi_{\nu} O_{\nu} \cup O_{\nu} \times O_{\nu}^{\times} \times \pi_{\nu} O_{\nu}
$$

Therefore as in the proof of Lemma $2.4 M_{\nu} \mathscr{F}_{0, \nu}=M_{\nu} \mathscr{F}_{1, \nu}+\frac{2}{q_{\nu}+1} \Phi_{1, \nu}$.

An easy argument shows that $M_{\nu} \Psi_{j, \nu}=\frac{1}{q_{\nu}+1} \sum_{k \geq 2 j} \Phi_{2^{k}, \nu}$. Therefore by (2.20) and Lemma 2.8

$$
\begin{gathered}
\left(q_{\nu}+1\right) M_{\nu} \Psi_{1, \nu}^{*}=\frac{1-q_{\nu}^{-1}}{q_{\nu}^{3}}\left(-\pi_{\nu}^{-2} \cdot \Phi_{0, \nu}^{\left(2 m_{\nu}-1\right)}+q_{\nu}^{2} \Phi_{0, \nu}^{2\left(m_{\nu}-1\right)}\right)+\frac{1}{q_{\nu}^{3}} \pi_{\nu}^{-2} \cdot \Phi_{2^{2 m_{\nu}+1, \nu}} \\
\left(q_{\nu}+1\right) M_{\nu} \Psi_{j, \nu}^{*}=\frac{1-q_{\nu}^{-1}}{q_{\nu}^{j+2}}\left(-\pi_{\nu}^{-2} \cdot \Phi_{0, \nu}^{2\left(m_{\nu}-j+2\right)}+q_{\nu}^{2} \Phi_{0, \nu}^{2\left(m_{\nu}-j\right)}\right) \\
+\frac{1}{q_{\nu}^{j+2}} \sum_{k \geq 2\left(m_{\nu}-j+2\right)} \pi_{\nu}^{-2} \cdot \Phi_{2^{k}, \nu}
\end{gathered}
$$

for $2 \leq j \leq m_{\nu}$, and

$$
\begin{aligned}
\left(q_{\nu}+1\right) M_{\nu} \Psi_{m_{\nu}+1, \nu}^{*}= & \frac{1-q_{\nu}^{-1}}{q_{\nu}^{m_{\nu}+3}}\left(-\pi_{\nu}^{-2} \cdot \Phi_{0, \nu}^{2}+q_{\nu} \pi_{\nu}^{-1} \cdot \Phi_{0, \nu}^{2}-q_{\nu} \pi_{\nu}^{-1} \cdot \Phi_{0, \nu}+q_{\nu}^{2} \Phi_{0, \nu}\right) \\
& +\frac{2\left(1-q_{\nu}^{-1}\right)}{q_{\nu}^{m_{\nu}+2}} \pi_{\nu}^{-1} \cdot \Phi_{1, \nu}+\frac{1}{q_{\nu}^{m_{\nu}+3}} \sum_{k \geq 2} \pi_{\nu}^{-2} \cdot \Phi_{2^{k}, \nu}
\end{aligned}
$$

By a straightforward calculation

$$
Z_{2}\left(s,\left(q_{\nu}+1\right) M_{\nu} \Psi_{j, \nu}^{*}\right)=-\frac{2\left(1-q_{\nu}^{-2}\right) q_{\nu}^{-2\left(m_{\nu}-j\right) s}}{q_{\nu}^{j+1}\left(1-q_{\nu}^{-2 s}\right)}, j=1, \ldots, m_{\nu}+1
$$

For $i \leq m_{\nu}-j+1$

$$
Z_{2^{2 i}}\left(s,\left(q_{\nu}+1\right) M_{\nu} \Psi_{j, \nu}^{*}\right)=-\frac{2\left(1-q_{\nu}^{-2}\right) q_{\nu}^{-2\left(m_{\nu}-j-i\right) s}}{q_{\nu}^{j+1}\left(1-q_{\nu}^{-2 s}\right)},
$$


and for $i>m_{\nu}-j+1$, including $i=m_{\nu}+\frac{1}{2}$,

$$
Z_{2^{2 i}}\left(s,\left(q_{\nu}+1\right) M_{\nu} \Psi_{j, \nu}^{*}\right)=-\frac{\left(1-q_{\nu}^{-1}\right) q_{\nu}^{4 s}\left(1+q_{\nu}^{1-2 s}\right)}{q_{\nu}^{j+2}\left(-q_{\nu}^{-2 s}\right)}+\frac{q_{\nu}^{4 s}}{q_{\nu}^{j+2}} .
$$

Note that a separate calculation is required to determine $Z_{2^{2 i}}\left(s,\left(q_{\nu}+1\right) M_{\nu}\right.$. $\left.\Psi_{j, \nu}^{*}\right)$ when $j=m_{\nu}+1$ or $i=m_{\nu}-j+1$. The results, however, turn out to be the same as for all other $j$ or $i \leq\left(m_{\nu}-j\right)$ respectively.

Since $\Phi_{2^{2 j, \nu}}=\left(q_{\nu}+1\right) M_{\nu}\left(\Psi_{j, \nu}-\Psi_{j+1, \nu}\right), j=1, \ldots, m_{\nu}$, and $\Phi_{2^{2 m_{\nu}+1, \nu}}=\left(q_{\nu}+\right.$ 1) $M_{\nu} \Psi_{m_{\nu}+1, \nu}$, one can now easily compute $\Gamma_{i 2^{j}}^{\nu}(s)$ :

PROPOSITION 2.9.

$$
\Gamma_{22^{2 j}}^{\nu}(s)=\frac{2\left(1-q_{\nu}^{-2}\right) q_{\nu}^{-2\left(m_{\nu}-j-1\right) s}\left(1-q_{\nu}^{1-2 s}\right)}{q_{\nu}^{j+2}\left(1-q_{\nu}^{-2 s}\right)}, j=1, \ldots, m_{\nu},
$$

and

$$
\Gamma_{22^{2 m_{\nu}+1}}^{\nu}(s)=-\frac{2\left(1-q_{\nu}^{-2}\right) q_{\nu}^{2 s}}{q_{\nu}^{m_{\nu}+2}\left(1-q_{\nu}^{-2 s}\right)} .
$$

For $i \leq m_{\nu}-j$,

$$
\begin{gathered}
\Gamma_{2^{2 j} 2^{2 j}}^{\nu}(s)=\frac{\left(1-q_{\nu}^{-2}\right) q_{\nu}^{-2\left(m_{\nu}-j-1-i\right) s}\left(1-q_{\nu}^{1-2 s}\right)}{q_{\nu}^{j+2}\left(1-q_{\nu}^{-2 s}\right)}, \\
\Gamma_{2^{2\left(m_{\nu}-j+1\right)} 2^{2 j}}^{\nu}(s)=\frac{\left(1-q_{\nu}^{-1}\right) q_{\nu}^{4 s}\left(1-q_{\nu}^{2-2 s}\right)}{q_{\nu}^{j+3}\left(1-q_{\nu}^{-2 s}\right)}-\frac{q_{\nu}^{4 s}}{q_{\nu}^{j+3}} .
\end{gathered}
$$

For $i \geq m_{\nu}-j+2$, including $i=m_{\nu}+\frac{1}{2}$,

$$
\Gamma_{2^{2 i_{2} j}}^{\nu}(s)=\frac{\left(1-q_{\nu}^{-1}\right) q_{\nu}^{4 s}\left(1-q_{\nu}^{2-2 s}\right)}{q_{\nu}^{j+3}\left(1-q_{\nu}^{-2 s}\right)},
$$

and for all $i$

$$
\Gamma_{2^{2 i_{2} 2 m_{\nu}+1}}^{\nu}(s)=\frac{q_{\nu}^{4 s}\left(1-q_{\nu}^{2-2 s}\right)}{q_{\nu}^{m_{\nu}+4}\left(1-q_{\nu}^{-2 s}\right)} .
$$

Our list of local functional equation coefficients for zeta functions associated with the space of binary quadratic forms is now complete. 


\section{The remainder}

Our next task is to compute the remainder term $T_{\mathrm{x}_{s}}(s)$ in the functional equation in Theorem 1.2 .

Let $\mathrm{x}_{S}=\left(\mathrm{x}_{\nu}\right)_{\nu \in S}$. Then by (1.2) and (1.18)

$$
T_{\mathrm{x}_{S}}(s)=D_{K}^{\frac{1}{2}} \rho_{K}^{3} \frac{T\left(2 s, \Phi^{*}\right)-T(3-2 s, \Phi)}{\Pi_{\nu \in S} Z_{x_{\nu}}\left(\frac{3}{2}-s, \Phi_{\nu}\right)}
$$

where $\Phi=\Pi_{\nu \in S} \Phi_{\nu} \times \Pi_{\nu \notin S} \Phi_{0, \nu}$ and for each $\nu \in S \operatorname{Supp}\left(\Phi_{\nu}\right) \subset V_{\mathrm{x}_{\nu}}$. For a $\mathscr{K}_{A}$-invariant function $\Phi, T(s, \Phi)=\left.\frac{d}{d w} T\left(s, w, T_{2} \Phi\right)\right|_{w=0}$ where $\left(T_{2} \Phi\right)(t, u)=$ $\Phi(0, t, u)$ and $T(s, w, \Psi)$ is given by the integral (1.4).

$T(s, w, \Psi)$ has an Euler product: let

$$
\alpha_{\nu}\left(u_{\nu}\right)= \begin{cases}\left(1+\left|u_{\nu}\right|_{\nu}^{2}\right)^{\frac{1}{2}} & \text { if } \nu \in M_{\mathbf{R}}(K) ; \\ \left(1+\left|u_{\nu}\right|_{\nu}\right) & \text { if } \nu \in M_{\mathbf{C}}(K) ; \\ \sup \left(1,\left|u_{\nu}\right|_{\nu}\right) & \text { if } \nu \in M_{0}(K)\end{cases}
$$

and

$$
T_{\nu}\left(s, w, \Psi_{\nu}\right)=\int_{K_{\nu}^{\times}} \int_{K_{\nu}}\left|t_{\nu}\right|_{\nu}^{s} \Psi_{\nu}\left(t_{\nu}, t_{\nu} u_{\nu}\right) \alpha_{\nu}\left(u_{\nu}\right)^{w} d u_{\nu} d^{\times} t_{\nu}
$$

Then for $\Psi=\Pi_{\nu \in M(K)} \Psi_{\nu}$

$$
T(s, w, \Psi)=D_{K}^{-\frac{1}{2}} \rho_{K}^{-1} \prod_{\nu \in M(K)} T_{\nu}\left(s, w, \Psi_{\nu}\right)
$$

The following two lemmas are essential for out calculations;

Lemma 3.1.

$$
T_{\nu}\left(s, w, T_{2} \Phi_{0, \nu}\right)=\frac{1-q_{\nu}^{w-s}}{\left(1-q_{\nu}^{-s}\right)\left(1-q_{\nu}^{1+w-s}\right)} .
$$

For the proof of this lemma we refer the reader to [16], Proposition (2.8).

Lemma 3.2. For $\mathscr{K}_{\nu}$-invariant function $\Phi_{\nu}$

$$
T_{\nu}\left(2 s, 0, T_{2} \Phi_{\nu}\right)=Z_{1}\left(s, \Phi_{\nu}\right)
$$


Proof.

$$
\begin{aligned}
T_{\nu}\left(2 s, 0, T_{2} \Phi_{\nu}\right) & =\int_{K_{\nu}^{\times}} \int_{K_{\nu}}\left|t_{\nu}\right|_{\nu}^{2 s} \Phi_{\nu}\left(0, t_{\nu}, t_{\nu} u_{\nu}\right) d u_{\nu} d^{\times} t_{\nu} \\
& =\int_{K_{\nu}^{\times}} \int_{K_{\nu}}\left|t_{\nu}\right|_{\nu}^{2 s} \Phi_{\nu}\left(n\left(u_{\nu}\right) a\left(t_{\nu}\right) \cdot(0,1,0)\right) d u_{\nu} d^{\times} t_{\nu} \\
& =\int_{K_{\nu}^{\times}} \int_{K_{\nu}} \int_{\mathscr{K}_{\nu}}\left|t_{\nu}\right|_{\nu}^{2 s} \Phi_{\nu}\left(\kappa_{\nu} n\left(u_{\nu}\right) a\left(t_{\nu}\right) \cdot \mathrm{x}_{1}\right) d \kappa_{\nu} d u_{\nu} d^{\times} t_{\nu} .
\end{aligned}
$$

The last expression is clearly $Z_{1}\left(s, \Phi_{\nu}\right)$.

By Lemma 3.1

$$
T\left(s, w, T_{2} \Phi\right)=D_{K}^{-\frac{1}{2}} \rho_{K}^{-1} T_{S}\left(s, w, T_{2} \Phi\right) \frac{\zeta_{K, S}(s) \zeta_{K, S}(s-w-1)}{\zeta_{K, S}(s-w)}
$$

where

$$
T_{S}\left(s, w, T_{2} \Phi\right)=\prod_{\nu \in S} T_{\nu}\left(s, w, T_{2} \Phi_{\nu}\right)
$$

For a $\mathscr{K}_{\nu}$-invariant function $\Phi_{\nu}$ let

$$
T_{\nu}\left(s, \Phi_{\nu}\right)=\left.\frac{d}{d w} T_{\nu}\left(s, w, T_{2} \Phi_{\nu}\right)\right|_{w=0}
$$

Then

$$
\begin{aligned}
T(s, \Phi)=D_{K}^{-\frac{1}{2}} \rho_{K}^{-1} & {\left[T_{S}\left(s, 0, T_{2} \Phi\right) \zeta_{K, S}(s-1)\left(\frac{\zeta_{K, S}^{\prime}}{\zeta_{K, S}}(s)-\frac{\zeta_{K, S}^{\prime}}{\zeta_{K, S}}(s-1)\right)\right.} \\
+ & \left.\zeta_{K, S}(s-1) \sum_{\nu \in S} \prod_{\mu \in S, \mu \neq \nu} T_{\mu}\left(s, 0, T_{2} \Phi_{\mu}\right) T_{\nu}\left(s, \Phi_{\nu}\right)\right] .
\end{aligned}
$$

Thanks to Lemma 3.2 we can rewrite (3.8) as follows:

$$
\begin{gathered}
T(2 s, \Phi)=D_{K}^{-\frac{1}{2}} \rho_{K}^{-1}\left[\prod_{\nu \in S} Z_{1}\left(s, \Phi_{\nu}\right) \zeta_{K, S}(2 s-1)\left(\frac{\zeta_{K, S}^{\prime}}{\zeta_{K, S}}(2 s)-\frac{\zeta_{K, S}^{\prime}}{\zeta_{K, S}}(2 s-1)\right)\right. \\
\left.+\zeta_{K, S}(2 s-1) \sum_{\nu \in S} \prod_{\mu \in S, \mu \neq \nu} Z_{1}\left(s, \Phi_{\mu}\right) T_{\nu}\left(s, \Phi_{\nu}\right)\right] .
\end{gathered}
$$

As in Section $1.4 \Phi^{*}=D_{K}^{-\frac{3}{2}} \Pi_{\nu \in M(K)} \pi_{\nu}^{-n_{\nu}} \cdot \Phi_{\nu}^{*}$ where $n_{\nu}$ is the order of the different of $K$ at $\nu$. It is easy to see that $T_{\nu}\left(s, w, \pi_{\nu}^{-n} \cdot \Psi_{\nu}\right)=q_{\nu}^{n s} T_{\nu}\left(s, w, \Psi_{\nu}\right)$. Also if $\nu \nmid 2, \Phi_{0, \nu}^{*}=\Phi_{0, \nu}$. Therefore if $S$ contains all places of $K$ that lie over 2, 


$$
\begin{aligned}
T\left(2 s, \Phi^{*}\right)=D_{K}^{2 s-2} \rho_{K}^{-1} & {\left[\prod_{\nu \in S} Z_{1}\left(s, \Phi_{\nu}^{*}\right) \zeta_{K, S}(2 s-1)\left(\frac{\zeta_{K, S}^{\prime}}{\zeta_{K, S}}(2 s)-\frac{\zeta_{K, S}^{\prime}}{\zeta_{K, S}}(2 s-1)\right)\right.} \\
& \left.+\zeta_{K, S}(2 s-1) \sum_{\nu \in S} \prod_{\mu \in S, \mu \neq \nu} Z_{1}\left(s, \Phi_{\mu}^{*}\right) T_{\nu}\left(s, \Phi_{\nu}^{*}\right)\right] .
\end{aligned}
$$

We know that $Z_{1}\left(s, \Phi_{\nu}^{*}\right)=\Gamma_{11}^{\nu}(s) Z_{1}\left(\frac{3}{2}-s, \Phi_{\nu}\right)$. Let $\delta_{\mathrm{x}_{\nu}}=1$ if $\mathrm{x}_{\nu}=\mathrm{x}_{1}$ and 0 otherwise and let $\delta_{\mathrm{x}_{S}}=\Pi_{\nu \in S} \delta_{\mathrm{x}_{\nu}}$. Then

$$
\begin{aligned}
& T_{\mathrm{x}_{S}}(s)=\rho_{K}^{2}\left[\delta_{\mathrm{x}_{S}} D_{K}^{2 s-\frac{3}{2}} \prod_{\nu \in S} \Gamma_{11}^{\nu}(s) \zeta_{K, S}(2 s-1)\left(\frac{\zeta_{K, S}^{\prime}}{\zeta_{K, S}}(2 s)-\frac{\zeta_{K, S}^{\prime}}{\zeta_{K, S}}(2 s-1)\right)\right. \\
& \left.-\delta_{\mathrm{x}_{S}} \zeta_{K, S}(2-2 s)\left(\frac{\zeta_{K, S}^{\prime}}{\zeta_{K, S}}(3-2 s)-\frac{\zeta_{K, S}^{\prime}}{\zeta_{K, S}}(2-2 s)\right)+\sum_{\nu \in S} \delta_{\mathrm{x}_{S-\nu \nu}} C\left(s, \Phi_{\nu}\right)\right]
\end{aligned}
$$

where $\delta_{\mathrm{x}_{S-(\nu)}}=\Pi_{\nu \in S, \mu \neq \nu} \delta_{\mathrm{x}_{\mu}}$ and

$$
\begin{aligned}
& \quad C\left(s, \Phi_{\nu}\right)= \\
& \frac{D_{K}^{2 s-\frac{3}{2}} \zeta_{K, S}(2 s-1) \prod_{\mu \in S, \mu \neq \nu} \Gamma_{11}^{\mu}(s) T_{\nu}\left(2 s, \Phi_{\nu}^{*}\right)-\zeta_{K, S}(2-2 s) T_{\nu}\left(3-2 s, \Phi_{\nu}\right)}{Z_{\mathrm{x}_{\nu}}\left(\frac{3}{2}-s, \Phi_{\nu}\right)}
\end{aligned}
$$

The numerator in (3.12) can be considerably simplified. Observe that by (2.4) $\frac{\zeta_{K, S}(2 s-1)}{\zeta_{K, S}(2-2 s)}=\Pi_{\nu \notin S} \Gamma_{11}^{\nu}(s)$ and that $D_{K}^{2 s-\frac{3}{2}} \Pi_{\nu \in M(K)} \Gamma_{11}^{\nu}(s)=1$. Therefore

$$
C\left(s, \Phi_{\nu}\right)=\frac{\zeta_{K, S}(2-2 s)\left(\left(\Gamma_{11}^{\nu}(s)\right)^{-1} T_{\nu}\left(2 s, \Phi_{\nu}^{*}\right)-T_{\nu}\left(3-2 s, \Phi_{\nu}\right)\right)}{Z_{\mathrm{x}_{\nu}}\left(\frac{3}{2}-s, \Phi_{\nu}\right)}
$$

Equation (3.11) implies that

$$
\left(\Gamma_{11}^{\nu}(s)\right)^{-1} T_{\nu}\left(2 s, \Phi_{\nu}^{*}\right)-T_{\nu}\left(3-2 s, \Phi_{\nu}\right)=\sum_{\mathrm{x}_{\nu}} C_{\mathrm{x}_{\nu}}(s) Z_{\mathrm{x}_{\nu}}\left(\frac{3}{2}-s, \Phi_{\nu}\right),
$$

i.e. it is a distribution that has the invariance property (1.7) under the action of $G_{K_{\nu}}$ with $\frac{3}{2}-s$ in place of $s$. This fact can also be verified by a direct local calculation.

With the notation of (3.14) we have: if $S$ contains all places of $K$ that lie over 2 , 


$$
\begin{aligned}
T_{\mathrm{x}_{S}}(s)=\rho_{K}^{2} \zeta_{K, S}(2-2 s) & {\left[\delta_{\mathrm{x}_{S}}\left(\frac{\zeta_{K, S}^{\prime}}{\zeta_{K, S}}(2 s)-\frac{\zeta_{K, S}^{\prime}}{\zeta_{K, S}}(2 s-1)\right)\right.} \\
& \left.-\delta_{\mathrm{x}_{S}}\left(\frac{\zeta_{K, S}^{\prime}}{\zeta_{K, S}}(3-2 s)-\frac{\zeta_{K, S}^{\prime}}{\zeta_{K, S}}(2-2 s)\right)+\sum_{\nu \in S} \delta_{\mathrm{x}_{S-\{\nu\}}} C_{\mathrm{x}_{\nu}}(s)\right] .
\end{aligned}
$$

What we have to do now is to compute $C_{\mathrm{x}_{\nu}}(s)$.

As in Section 2, we will write $C_{i, \nu}(s)$ for $C_{\mathrm{x}_{\nu}}(s)$ if $\mathrm{x}_{\nu}$ is the standard orbital representative of the local orbit $V_{i}$. Clearly

$$
C_{i, \nu}(s)=\left(\Gamma_{11}^{\nu}(s)\right)^{-1} T_{\nu}\left(2 s, \Phi_{i, \nu}^{*}\right)-T_{\nu}\left(3-2 s, \Phi_{i, \nu}\right) .
$$

If $\nu \nmid 2$, the right hand side of (3.16) is rather easy to compute All we need is the following lemma:

LEMMA 3.3.

$$
T_{\nu}\left(s, \Phi_{0, \nu}\right)=\frac{q_{\nu}^{1-s}\left(1-q_{\nu}^{-1}\right) \log q_{\nu}}{\left(1-q_{\nu}^{1-s}\right)^{2}\left(1-q_{\nu}^{-s}\right)},
$$

and for any $i$ in the index set of local $G_{K_{\nu}}$ orbits $T_{\nu}\left(s, \Phi_{i, \nu}\right)=0$.

Proof. The first statement follows from Lemma 3.1 by differentiation. As for the second, one only need to observe that $T_{\nu}\left(s, w, \Phi_{i, \nu}\right)=\left\{\begin{array}{ll}0 & \text { if } i \neq 1 \\ 1 & \text { if } i=1\end{array}\right.$.

The Fourier transforms $\Phi_{i, \nu}^{*}, i=1,2,2(j)$, are given in $(2.10),(2.12)$ and (2.15). A quick calculation now shows:

Proposition 3.4.

$$
\begin{gathered}
C_{1, \nu}(s)=-\log q_{\nu} \frac{q_{\nu}^{2-2 s}\left(1-q_{\nu}^{-1}\right)^{2}\left(1+q_{\nu}^{1-2 s}\right)}{2\left(1-q_{\nu}^{2-2 s}\right)\left(1-q_{\nu}^{1-2 s}\right)\left(1-q_{\nu}^{-2 s}\right)}, \\
C_{2, \nu}(s)=\log q_{\nu} \frac{q_{\nu}^{2-2 s}\left(1-q_{\nu}^{-1}\right)^{2}}{2\left(1-q_{\nu}^{2-2 s}\right)\left(1-q_{\nu}^{-2 s}\right)}
\end{gathered}
$$

and

$$
C_{2(j), \nu}(s)=\log q_{\nu} \frac{\left(1-q_{\nu}^{-1}\right)^{2}\left(1+q_{\nu}^{1-2 s}\right)}{2\left(1-q_{\nu}^{2-2 s}\right)\left(1-q_{\nu}^{-2 s}\right)} .
$$


To compute $C_{i, \nu}(s)$ for $\nu \mid 2$ we need

LEMMA 3.5. For a non-negative integer $j$

$$
T_{\nu}\left(s, w, T_{2} \Phi_{0, \nu}^{j}\right)=\frac{q_{\nu}^{-\tilde{j} s}}{1-q_{\nu}^{-s}} \frac{q_{\nu}^{-2}\left(1-q_{\nu}^{-w}\right)}{1-q_{\nu}^{-1-w}}+\frac{q_{\nu}^{\tilde{j}(w+1-s)}\left(1-q_{\nu}^{-1}\right)}{\left(1-q_{\nu}^{w+1-s}\right)\left(1-q_{\nu}^{-1-w}\right)},
$$

and

$T_{\nu}\left(s, \Phi_{0, \nu}^{j}\right)=\frac{q_{\nu}^{-\tilde{j} s}}{1-q_{\nu}^{-s}} \frac{\log q_{\nu}}{q_{\nu}-1}-\frac{q_{\nu}^{\tilde{j}(1-s)} \log q_{\nu}\left(1-q_{\nu}^{2-s}\right)}{\left(q_{\nu}-1\right)\left(1-q_{\nu}^{1-s}\right)^{2}}+\frac{\tilde{j}_{\nu}^{\tilde{j}(1-s)} \log q_{\nu}}{1-q_{\nu}^{1-s}}$

where $\tilde{j}=\left\{\begin{array}{ll}\frac{j}{2} & \text { if } j \text { is even } \\ \frac{j+1}{2} & \text { if } j \text { is odd }\end{array}\right.$.

Proof.

$$
\begin{aligned}
T_{\nu}(s, & \left.w, T_{2} \Phi_{0, \nu}^{j}\right)=\int_{t_{\nu} \in \pi_{\nu}^{\tilde{j}} O_{\nu}} \int_{t_{\nu} u_{\nu} \in O_{\nu}}\left|t_{\nu}\right|_{\nu}^{s} \sup \left(1,\left|u_{\nu}\right|_{\nu}\right)^{w} d u_{\nu} d^{I} t_{\nu} \\
= & \int_{t_{\nu} \in \pi_{\nu}^{\tilde{j}} O_{\nu}}\left|t_{\nu}\right|_{\nu}^{s}\left(1+\sum_{n=1}^{\mathrm{ord} t_{\nu}} q_{\nu}^{n w} q_{\nu}^{n}\left(1-q_{\nu}^{-1}\right)\right) d^{\times} t_{\nu} \\
= & \int_{t_{\nu} \in \pi_{\nu}^{\tilde{j}} O_{\nu}}\left(\left|t_{\nu}\right|_{\nu}^{s} \frac{q_{\nu}^{-1}-q_{\nu}^{-1-w}}{1-q_{\nu}^{-1-w}}+\left|t_{\nu}\right|_{\nu}^{s-w-1} \frac{1-q_{\nu}^{-1}}{\left(1-q_{\nu}^{w+1-s}\right)\left(1-q_{\nu}^{-1-w}\right)}\right) d^{\times} t_{\nu} \\
= & \frac{q_{\nu}^{-\tilde{j} s}}{1-q_{\nu}^{-s}} \frac{q_{\nu}^{-1}\left(1-q_{\nu}^{-w}\right)}{1-q_{\nu}^{-1-w}}+\frac{q_{\nu}^{\tilde{j}(w+1-s)}\left(1-q_{\nu}^{-1}\right)}{\left(1-q_{\nu}^{w+1-s}\right)\left(1-q_{\nu}^{-1-w}\right)}
\end{aligned}
$$

as claimed. The formula for $T_{\nu}\left(s, \Phi_{0, \nu}^{j}\right)$ can now be obtained by straightforward differentiation.

Using (2.17), (2.19) and (2.22)-(2.24) we can now find $C_{i, \nu}(s)$. We spare the reader a tedious calculation and just list the final result here.

Proposition 3.6. For $\nu \mid 2$

$$
C_{1, \nu}(s)=\log q_{\nu}\left[-\frac{\left(1-q_{\nu}^{2-2 s}\right)}{\left(q_{\nu}-1\right)\left(1-q_{\nu}^{1-2 s}\right)}+m_{\nu}\right.
$$




$$
\begin{aligned}
& \left.-\frac{\left(1+q_{\nu}^{2-2 j}\right)}{2\left(1-q_{\nu}^{2-2 s}\right)}+\frac{|2|_{\nu}\left(1+q_{\nu}^{-1}\right)\left(1-q_{\nu}^{1-2 s}\right)^{2}}{2\left(1-q_{\nu}^{-1}\right)\left(1-q_{\nu}^{-2 s}\right)\left(1-q_{\nu}^{2-2 s}\right)}\right], \\
& C_{2, \nu}(s)=\log q_{\nu}\left[-\frac{1}{2}+\frac{|2|_{\nu}\left(1-q_{\nu}^{1-2 s}\right)^{2}}{2\left(1-q_{\nu}^{-2 s}\right)\left(1-q_{\nu}^{2-2 s}\right)}\right],
\end{aligned}
$$

for $j=1, \ldots, m_{\nu}$

$$
C_{2^{2 j, \nu}}(s)=\log q_{\nu}\left[-2\left(1-q_{\nu}^{-1}\right) q_{\nu}^{j(2 s-2)}+\frac{|2|_{\nu}\left(1-q_{\nu}^{-2}\right) q_{\nu}^{j(2 s-1)}\left(1-q_{\nu}^{1-2 s}\right)^{2}}{\left(1-q_{\nu}^{-2 s}\right)\left(1-q_{\nu}^{2-2 s}\right)}\right],
$$

and

$$
\begin{aligned}
C_{2^{2 m_{\nu+1}, \nu}}(s)=\mid & \left.2\right|_{\nu} ^{2-2 s} \log q_{\nu}\left[-2 q_{\nu}^{-1}+\frac{\left(1-q_{\nu}^{-1}\right)\left(1-q_{\nu}^{1-2 s}\right)}{1-q_{\nu}^{2-2 s}}\right. \\
& \left.+\frac{q_{\nu}^{-1}\left(1+q_{\nu}^{-1}\right)\left(1-q_{\nu}^{1-2 s}\right)^{2}}{\left(1-q_{\nu}^{-2 s}\right)\left(1-q_{\nu}^{2-2 s}\right)}\right]
\end{aligned}
$$

It remains to compute $C_{i, \nu}(s)$ for archimedean places. Before we do this though, we need to make a slight correction to equations (3.10)-(3.15). As written, these equations are only valid if $S$ contains all places of $K$ that lie over 2. To make $(3.10)-(3.12)$ valid for all $S$ one needs to replace $\zeta_{K, S}(2 s-1)$ by $\Pi_{\nu \notin S} T_{\nu}\left(2 s, 0, T_{2} \Phi_{0, \nu}^{*}\right)$ and $\frac{\zeta_{K, S}^{\prime}}{\zeta_{K, S}}(2 s)-\frac{\zeta_{K, S}^{\prime}}{\zeta_{K, S}}(2 s-1)$ by the logarithmic derivative of $\Pi_{\nu \notin S} T_{\nu}\left(s, w, T_{2} \Phi_{0, \nu}^{*}\right)$ at $w=0$. By Lemma $3.2 T_{\nu}\left(s, 0, T_{2} \Phi_{0, \nu}^{*}\right)=Z_{1}\left(s, \Phi_{0, \nu}^{*}\right)$, so when one pulls out $\zeta_{K, S}$ in $(3.12)$ one has $\Pi_{\nu \notin S} \frac{Z_{1}\left(s, \Phi_{0, \nu}^{*}\right)}{Z_{1}\left(\frac{3}{2}-s, \Phi_{0, \nu}\right)}=\Pi_{\nu \notin S} \Gamma_{11}^{\nu}(s)$.

Thus (3.13) and (3.14) are valid for any $S$.

$\Phi_{0, \nu}^{*}=\Phi_{0, \nu}^{2 m_{\nu}}$, and we can compute the logarithmic derivative of $T_{\nu}(s, w$, $\left.T_{2} \Phi_{0, \nu}^{*}\right)$ at $w=0$ using Lemma 3.5. Comparing with the logarithmic derivative of $T_{\nu}\left(s, w, T_{2} \Phi_{0, \nu}\right)$ at $w=0$ we obtain

$$
\begin{aligned}
& \left.\frac{d}{d w} \log T_{\nu}\left(s, w, T_{2} \Phi_{0, \nu}^{*}\right)\right|_{w=0}=\left.\frac{d}{d w} \log T_{\nu}\left(s, w, T_{2} \Phi_{0, \nu}\right)\right|_{w=0} \\
& \quad+m_{\nu} \log q_{\nu}-\log q_{\nu} \frac{q_{\nu}^{-1}\left(1-q_{\nu}^{-m_{\nu}}\right)\left(1-q_{\nu}^{1-2 s}\right)}{\left(1-q_{\nu}^{-1}\right)\left(1-q_{\nu}^{-2 s}\right)} .
\end{aligned}
$$

Therefore the version of (3.15) that is valid for any $S$ is

$$
T_{\mathrm{x}_{S}}(s)=\rho_{K}^{2} \zeta_{K, S}(2-2 s)\left[\delta _ { \mathrm { x } _ { S } } \left\{\sum_{\nu \notin S}\left(-\log |2|_{\nu}-\log q_{\nu} \frac{q_{\nu}^{-1}\left(1-|2|_{\nu}\right)\left(1-q_{\nu}^{1-2 s}\right)}{\left(1-q_{\nu}^{-1}\right)\left(1-q_{\nu}^{-2 s}\right)}\right)\right.\right.
$$




$$
\begin{gathered}
\left.+\left(\frac{\zeta_{K, S}^{\prime}}{\zeta_{K, S}}(2 s)-\frac{\zeta_{K, S}^{\prime}}{\zeta_{K, S}}(2 s-1)\right)-\left(\frac{\zeta_{K, S}^{\prime}}{\zeta_{K, S}}(3-2 s)-\frac{\zeta_{K, S}^{\prime}}{\zeta_{K, S}}(2-2 s)\right)\right\} \\
\left.+\sum_{\nu \in S} \delta_{\mathrm{x}_{S-\langle\nu\rangle}} C_{\mathrm{x}_{\nu}}(s)\right] .
\end{gathered}
$$

We now specialize to $K=\mathbf{Q}$ and $S=\{\infty\}$. In this case (3.18) reads:

$$
\begin{aligned}
T_{\mathrm{x}_{1}}(s) & =\zeta(2-2 s)\left[\frac{\log 2}{2\left(1-2^{-2 s}\right)}+\left(\frac{\zeta^{\prime}}{\zeta}(2 s)-\frac{\zeta^{\prime}}{\zeta}(2 s-1)\right)\right. \\
& \left.-\left(\frac{\zeta^{\prime}}{\zeta}(3-2 s)-\frac{\zeta^{\prime}}{\zeta}(2-2 s)\right)+C_{1, \infty}(s)\right]
\end{aligned}
$$

and

$$
T_{\mathrm{x}_{2}}(s)=\zeta(2-2 s) C_{2, \infty}(s) .
$$

By [3], Theorem 0.2 , when $K=\mathbf{Q}$ and $S=\{\infty\} \xi_{\mathrm{x}_{1}}(s)=\xi_{+}(s)$ and $\xi_{\mathrm{x}_{2}}(s)=$ $\pi \xi_{-}(s)$ where $\xi_{ \pm}(s)$ are the Dirichlet series of Shintani [12]. Thanks to [12], Theorem 2, we know what the remainder is in this case (see $(0.1)$ ). Comparing (3.19) and (3.20) with (0.1) we obtain:

Proposition 3.7. Let $\nu$ be a real place of $K$. Then

$$
C_{1, \nu}(s)=\frac{1}{2}\left(\frac{\Gamma^{\prime}}{\Gamma}(s)-\frac{\Gamma^{\prime}}{\Gamma}\left(s-\frac{1}{2}\right)\right)
$$

and

$$
C_{2, \nu}(s)=\frac{-\pi}{2 \sin \pi s}
$$

It would be interesting to obtain $C_{i, \nu}(s), i=1,2$, for a real place $\nu$ of $K$ directly without referring to the work of Shintani. I leave this project, however, to those readers who are more profficient in real analysis than I am.

Finally, let $\nu$ be a complex place of $K$. Let $\Phi(x)=e^{-\pi\left(2\left|x_{1}\right| \mathrm{c}+\left|x_{2}\right| \mathrm{c}+2\left|x_{3}\right|_{c}\right)}$ where $|x|_{\mathbf{C}}$ here denotes the complex modulus of $x$. The function $\Phi(x)$ is $\mathscr{K}_{\mathbf{C}}$-invariant. Moreover, $\Phi^{*}=2 \Phi$. By $(2.2)$

$$
\begin{aligned}
Z_{1}(s, \Phi) & =\int_{\mathbf{C}^{\times}} \int_{\mathbf{C}}|t|_{\mathbf{C}}^{2 s-1} e^{-\pi\left(|t|_{\mathbf{C}}+2|u|_{\mathbf{C}}\right)} d^{\times} t d u \\
& =(2 \pi)^{2} \int_{0}^{\infty} \int_{0}^{\infty} \lambda^{2 s-1} e^{-\pi(\lambda+2 \mu)} d^{\times} \lambda d \mu=2 \pi^{2-2 s} \Gamma(2 s-1) .
\end{aligned}
$$




$$
\begin{aligned}
T_{\nu}\left(2 s, w, T_{2} \Phi\right) & =\int_{\mathbf{C}^{\times}} \int_{\mathbf{C}}|t|_{\mathbf{C}}^{2 s} e^{-\pi\left(|t|_{\mathbf{C}}^{\left(1+2|u|_{\mathbf{C}}\right)}\right.}\left(1+|u|_{\mathbf{C}}\right)^{w} d u d^{\times} t \\
& =(2 \pi)^{2} \int_{0}^{\infty} \int_{0}^{\infty} \lambda^{2 s} e^{-\pi(1+2 \mu)}(1+\mu)^{w} d \mu d^{\times} \lambda \\
& =4 \pi^{2-2 s} \Gamma(2 s) \int_{0}^{\infty}(1+2 \mu)^{-2 s}(1+\mu)^{w} d \mu .
\end{aligned}
$$

The last integral can be evaluated in terms of hypergeometric functions. In fact, it equals $2^{-2 s} \frac{F\left(2 s, 2 s-w-1 ; 2 s-w ; \frac{1}{2}\right)}{2 s-w-1}$. We, however, are only interested in its derivative with respect to $w$ at $w=0$. Differentiating, we obtain

$$
\begin{aligned}
T_{\nu}(2 s, \Phi) & =4 \pi^{2-2 s} \Gamma(2 s) \int_{0}^{\infty}(1+2 \mu)^{-2 s} \log (1+\mu) d \mu \\
& =\frac{2 \pi^{2-2 s} \Gamma(2 s)}{2 s-1} \int_{0}^{\infty}(1+2 \mu)^{-2 s+1}(1+\mu)^{-1} d \mu
\end{aligned}
$$

Set $\tau=(1+2 \mu)^{-1}$. Then

$$
\begin{aligned}
T_{\nu}(2 s, \Phi) & =2 \pi^{2-2 s} \Gamma(2 s-1) \int_{0}^{1} \frac{\tau^{2 s-2}}{(1+\tau)} d \tau \\
& =2 \pi^{2-2 s} \Gamma(2 s-1) \sum_{n=0}^{\infty} \frac{1}{2 s-2+n}(-1)^{n} \\
& =\pi^{2-2 s} \Gamma(2 s-1)\left(\frac{\Gamma^{\prime}}{\Gamma}\left(s-\frac{1}{2}\right)-\frac{\Gamma^{\prime}}{\Gamma}(s-1)\right) .
\end{aligned}
$$

Substituting (3.24) and (3.21) in (3.14) we obtain:

Proposition 3.8. If $\nu$ is a complex place of $K$,

$$
C_{1, \nu}(s)=\frac{1}{2}\left(\frac{\Gamma^{\prime}}{\Gamma}\left(s-\frac{1}{2}\right)-\frac{\Gamma^{\prime}}{\Gamma}(s-1)\right)-\frac{1}{2}\left(\frac{\Gamma^{\prime}}{\Gamma}(1-s)-\frac{\Gamma^{\prime}}{\Gamma}\left(\frac{1}{2}-s\right)\right) .
$$

We summarize our results in the theorem below:

THEOREM 3.9. The Dirichlet series $\xi_{\mathrm{x}_{s}}(s), \xi_{\mathrm{x}_{s}}^{*}(s)$ satisfy a functional equation

$$
\xi_{\mathrm{x}_{s}}\left(\frac{3}{2}-s\right)=D_{K}^{2 s-\frac{3}{2}} \sum_{\mathrm{y}_{s}} \Gamma_{\mathrm{x}_{\mathrm{y}_{\mathrm{s}}}}(s) \xi_{\mathrm{y}_{s}}^{*}(s)+T_{\mathrm{x}_{s}}(s)
$$

The functional equation coefficients $\Gamma_{\mathrm{x}_{s} \mathrm{y}_{s}}(s)=\Pi_{\nu \in S} \Gamma_{\mathrm{x}_{\nu} \mathrm{y}_{\nu}}(s)$, and the values of 
$\Gamma_{\mathrm{x}_{\nu} \mathrm{y}_{\nu}}(s)$ are given in Propositions 2.2, 2.3, 2.5, 2.7 and 2.9. The remainder $T_{\mathrm{x}_{S}}=0$ if $\mathrm{x}_{S}$ is non-split in at least tuo places $\nu \in S$. If $\mathrm{x}_{S}$ is non-split at just one place $\nu \in S$,

$$
T_{\mathrm{x}_{S}}(s)=\rho_{K}^{2} \zeta_{K, S}(2-2 s) C_{\mathrm{x}_{\nu}}(s),
$$

where the values of $C_{\mathrm{x}_{\nu}}(s)$ are given in Propositions 3.4, 3.6, 3.7 and 3.8. If $\mathrm{x}_{S}$ is split at all $\nu \in S$,

$$
\begin{gathered}
T_{\mathrm{x}_{S}}(s)=\rho_{K}^{2} \zeta_{K, S}(2-2 s)\left[\sum_{\nu \notin S}\left(-\log |2|_{\nu}-\log q_{\nu} \frac{q_{\nu}^{-1}\left(1-|2|_{\nu}\right)\left(1-q_{\nu}^{1-2 s}\right)}{\left(1-q_{\nu}^{-1}\right)\left(1-q_{\nu}^{-2 s}\right)}\right)\right. \\
+\left(\frac{\zeta_{K, S}^{\prime}}{\zeta_{K, S}}(2 s)-\frac{\zeta_{K, S}^{\prime}}{\zeta_{K, S}}(2 s-1)\right)-\left(\frac{\zeta_{K, S}^{\prime}}{\zeta_{K, S}}(3-2 s)-\frac{\zeta_{K, S}^{\prime}}{\zeta_{K, S}}(2-2 s)\right) \\
\left.+\sum_{\nu \in S} C_{1, \nu}(s)\right] .
\end{gathered}
$$

\section{Dirichlet series with a modular functional equation}

In this section we are going to construct a family of Dirichlet series $\xi(s)=$ $\sum_{n} a(n) \lambda_{n}^{-s}$, where $\lambda_{n}$ are rational numbers with bounded denominators, that satisfy

$$
\left(\frac{\pi}{2}\right)^{s-\frac{3}{2}} \Gamma\left(\frac{3}{2}-s\right) \xi\left(\frac{3}{2}-s\right)=\left(\frac{\pi}{2}\right)^{-s} \Gamma(s) \xi(s) .
$$

We specialize to $K=\mathbf{Q}$. Take $\Phi=\Pi_{\nu \in M(\mathbf{Q})} \Phi_{\nu}$ where two of the $\Phi_{\nu}$ satisfy $Z_{1}\left(s, \Phi_{\nu}\right)=0$. Then by Proposition $2.2 Z_{1}\left(s, \Phi_{\nu}^{*}\right)=0$, and by Lemma 3.2 and (3.8) $T\left(2 s, \Phi^{*}\right)=T(3-2 s, \Phi)=0$. The functional equation (1.2) now takes a particularly simple form:

$$
Z\left(\frac{3}{2}-s, \Phi\right)=Z\left(s, \Phi^{*}\right)
$$

Let $H_{\infty}=H_{\mathbf{R}}$ and $H(\infty)=H_{\infty} \times \Pi_{p} H_{\mathbf{z}_{p}}$. Then $H_{A}=H(\infty) H_{\mathbf{Q}}$. Moreover, the measure $d h$ on $H_{A}$ equals $d h_{\infty} \times \Pi_{p} d h_{p}$.

Write $\Phi=\Phi_{\infty} \times \Phi_{0}$ where $\Phi_{0}=\Pi_{p} \Phi_{p}$. By normalizing if necessary we may assume that all $\Phi_{p}$ are $H_{\mathbf{Z}_{p}}$-invariant. Then

$$
\begin{aligned}
Z(s, \Phi) & =\int_{H_{\infty} / H_{\mathbf{Z}}}\left|\chi\left(h_{\infty}\right)\right|^{s} \sum_{x \in V_{\mathbf{Q}}^{\prime \prime}} \Phi_{\infty}\left(h_{\infty} \cdot x\right) \Phi_{0}(x) d h_{\infty} \\
& =\sum_{x \in H_{\mathbf{Z}} \backslash V_{\mathbf{Q}}^{\prime \prime}} \Phi_{0}(x) \int_{H_{\infty} /\left(H_{x}\right)_{\mathbf{Z}}}\left|\chi\left(h_{\infty}\right)\right|^{s} \Phi_{\infty}\left(h_{\infty} \cdot x\right) d h_{\infty} .
\end{aligned}
$$

The last integral was evaluated in [3], Equation 7.6. It equals 


$$
c_{x} \mu_{\infty}(x)|P(x)|^{-s} Z_{x}\left(s, \Phi_{\infty}\right)
$$

where

$$
c_{x}= \begin{cases}1 & \text { if }\left(H_{x}\right)_{\mathbf{z}}=\left(H_{x}^{0}\right)_{\mathbf{z}} \\ \frac{1}{2} & \text { otherwise }\end{cases}
$$

and

$$
\mu_{x}= \begin{cases}\frac{4 \pi}{\omega_{x}} & \text { if } x \text { is definite; } \\ 2 \log \varepsilon_{x} & \text { if } x \text { is indefinite }\end{cases}
$$

Here $\omega_{x}$ is the number of automorphs of $x$ if $x$ is definite, and $\varepsilon_{x}$ is the fundamental unit that generates the group of automorphs of $x$ if $x$ is indefinite.

Let $\left(V_{1}\right)_{\mathbf{Q}}$ denote the set of indefinite binary quadratic forms with coefficients in $\mathbf{Q}$ that do not split over $\mathbf{Q}$, and $\left(V_{2}\right)_{\mathbf{Q}}$ the set of definite binary quadratic forms with coefficients in $\mathbf{Q}$. Then we have

$$
Z(s, \Phi)=Z_{1}\left(s, \Phi_{\infty}\right) \xi_{1}\left(s, \Phi_{0}\right)+Z_{2}\left(s, \Phi_{\infty}\right) \xi_{2}\left(s, \Phi_{0}\right)
$$

where

$$
\xi_{i}\left(s, \Phi_{0}\right)=\sum_{x \in H_{\mathbf{Z}} \backslash\left(V_{i}\right) \mathbf{Q}} c_{x} \mu_{\infty}(x) \Phi_{0}(x)|P(x)|^{-s} .
$$

The series $\xi_{2}\left(s, \Phi_{0}\right)$ can be described in more classical terms. Let $V_{2}^{+}$denote the set of positive definite binary quadratic forms. Since $H_{\mathbf{Z}}=\left\{ \pm 1, P G L_{2}(\mathbf{Z})\right.$ ), any form in $\left(V_{2}\right)_{\mathbf{Q}}$ is $H_{Z}$-equivalent to a form in $\left(V_{2}^{+}\right)_{\mathbf{Q}}$. Moreover, $c_{x}=\frac{1}{2}$ if $H_{\mathbf{Z}^{-}}$orbit of $x$ coincides with $S L_{2}(\mathbf{Z})$-orbit of $x$ and $c_{x}=1$ if $H_{\mathbf{Z}^{-}}$orbit of $x$ decomposes into two $S L_{2}(\mathbf{Z})$-orbits. Hence

$$
\xi_{2}\left(s, \Phi_{0}\right)=2 \pi \sum_{x \in S L_{2}(\mathbf{Z}) \backslash\left(V_{2}^{+}\right)_{\mathbf{Q}}} \frac{\Phi_{0}(x)}{\omega_{x}}|P(x)|^{-s} .
$$

Now let $\operatorname{Supp}\left(\Phi_{\infty}\right) \subset\left(V_{2}\right)_{\mathbf{R}}$. Then $Z_{1}\left(s, \Phi_{\infty}^{*}\right)=Z_{1}\left(\frac{3}{2}-s, \Phi_{\infty}\right)=0$, and by (1.11) and Proposition 2.3

$$
Z_{2}\left(S, \Phi_{\infty}^{*}\right)=2^{2 s-1} \pi^{\frac{1}{2}-2 s} \Gamma\left(s-\frac{1}{2}\right) \Gamma(s) \cos (\pi s) Z_{2}\left(\frac{3}{2}-s, \Phi_{\infty}\right) .
$$

This combined with (4.2) yields 
Proposition 4.1. Let $\Phi_{0}=\Pi_{p} \Phi_{p}$ be a product of locally constant functions with compact support on $V_{\mathbf{Q}_{p}}$ such that for almost all $p \Phi_{p}$ is the characteristic function of $V_{\mathbf{Z}_{p}}$ and for one of the primes $p Z_{1}\left(s, \Phi_{p}\right)=0$. Let

$$
L\left(s, \Phi_{0}\right)=\left(\frac{\pi}{2}\right)^{-s} \Gamma(s) \xi_{2}\left(s, \Phi_{0}\right)
$$

where $\xi_{2}\left(s, \Phi_{0}\right)$ is given by (4.7). Then

$$
L\left(\frac{3}{2}-s, \Phi_{0}\right)=-\sqrt{2} L\left(s, \Phi_{0}^{*}\right)
$$

The Fourier transform $\Phi_{0}^{*}=\Pi_{p} \Phi_{p}^{*}$. The local measures $d x_{p}$ are self-dual for all $p \neq 2$, and for $p=2\left(\Phi_{2}^{*}\right)^{*}=\frac{1}{2} \Phi_{2}$. Therefore we can pick $\Phi_{p}$ so that $\Phi_{0}^{*}=$ $\frac{C}{\sqrt{2}} \Phi_{0}$ where $C= \pm 1$. We now have

Corollary 4.2. Suppose $\Phi_{0}$ satisfies the hypotheses of Proposition 4.1 and furthermore $\Phi_{0}^{*}=\frac{C}{\sqrt{2}} \Phi_{0}$ where $C= \pm 1$. Then

$$
L\left(\frac{3}{2}-s, \Phi_{0}\right)=-C L\left(s, \Phi_{0}\right)
$$

We conclude this paper by giving an example of a series satisfying equation (4.1). Let $q$ be an odd prime. Set

1) $\Phi_{2}=\Phi_{0,2}-\sqrt{2} \Phi_{0,2}^{*}$;

2) $\Phi_{q}=\Phi_{2, q}+\Phi_{2, q}^{*}$;

3) $\Phi_{p}=\Phi_{0, p}$ if $p$ is an odd prime other than $q$.

Then $\Phi_{0}=\Pi_{p} \Phi_{p}$ satisfies the hypotheses of Corollary 4.2 with $C=-1$, and hence $\xi_{2}\left(s, \Phi_{0}\right)$ satisfies equation (4.1).

The function $\Phi_{2, q}^{*}$ is given in (2.12). Note that $\Phi_{1, q}+\Phi_{2, q}$ is the characteristic function of the set of forms with $\mathbf{Z}_{q}$-integral coefficients whose discriminants are $\mathbf{Z}_{q}$-units and $\Phi_{2, q}$ the characteristic function of the set of forms with $\mathbf{Z}_{q}$-integral coefficients whose discriminants are non-squares in $\mathbf{Z}_{q}^{\times}$.

Let $H(n)$ be the number of $S L_{2}(\mathbf{Z})$-classes of positive definite integral binary quadratic forms of discriminant $-n$, counted with multiplicities as in (0.4). Then (2.12) and (4.7) imply that 


$$
\begin{aligned}
\frac{2}{\pi} \xi_{2}\left(s, \Phi_{0}\right)= & \left(1-q^{-1}\right)\left(1-q^{2 s-1}\right) \sum_{n=1}^{\infty}\left(\frac{H(n)}{n^{s}}-\sqrt{2} \frac{H(4 n)}{(4 n)^{s}}\right) \\
& +q^{2 s-1}\left(1-q^{-1}\right) \sum_{n=1,(n, q)=1}^{\infty}\left(\frac{H(n)}{n^{s}}-\sqrt{2} \frac{H(4 n)}{(4 n)^{s}}\right) \\
& +2\left(1+q^{2 s-2}\right) \sum_{n \text { non-sq. (mod } q)}\left(\frac{H(n)}{n^{s}}-\sqrt{2} \frac{H(4 n)}{(4 n)^{s}}\right) .
\end{aligned}
$$

The functional equation for the series (4.9) suggests that the inverse Mellin transform of $\left(2 \pi q^{2}\right)^{-s} \Gamma(s) \xi_{2}\left(s, \Phi_{0}\right)$ plus an appropriate constant term (necessary since $\left(2 \pi q^{2}\right)^{-s} \Gamma(s) \xi_{2}\left(s, \Phi_{0}\right)$ has a simple pole at $\left.s=3 / 2\right)$ is a modular form of weight $3 / 2$ on $\Gamma_{0}\left(16 q^{4}\right)$. It is indeed so as can be easily seen from Theorem 3.3 and Corollary 3.4 of [1].

The results of this section point to a curious connection between modular forms of weight $3 / 2$ and zeta functions associated with the space of binary quadratic forms. It would be extremely interesting to investigate this connection further.

\section{REFERENCES}

[1] Cohen, H., Sums involving the values at negative integers of $L$-functions of quadratic characters, Math. Ann., 217, (1975), 271-285.

[2] Datskovsky, B., On zeta functions associated with the space of binary cubic forms with coefficients in a function field, Ph. D. thesis, Harvard University, 1984.

[3] Datskovsky, B., A mean-value theorem for class numbers of quadratic extensions, in A Tribute to Emil Grosswald: Number Theory and Related Analysis, Contemporary Mathematics, vol. 143, Amer. Math. Soc., 1993, 179-242.

[4] Datskovsky, B. and Wright, D. J., The adelic zeta function associated with the space of binary cubic forms, II; Local theory, J. Reine Angew. Math., 367, (1986), $27-75$.

[5] Igusa, J.-I., On functional equations of complex powers, Invent Math., 85, (1986), $1-29$.

[6] Muller, I., Décomposition orbitale des espaces préhomogènes régulieres de type parabolique commutatif et application, C. R. Acad. Sc. Paris, 303 (1986), 495-498.

[ 7 ] Rallis, S., and Schiffmann, G., Distributions invariantes par le groupe orthogonal, in Analyse Harmonique sur les Groupes de Lie, Lecture Notes in Mathematics 497, Springer-Verlag, Berlin, Heidelberg, New York, 1975, pp. 494-642.

[8] Saito, $H .$, On $L$-functions associated with the vector space of binary quadratic forms, Nagoya Math. J., 130 (1993), 140-176.

[9] Sato, F., On functional equations of zeta distributions, in Automorphic Forms and Geometry of Arithmetic Varieties. Advanced Studies in Pure Mathematics 15, Academic Press, New York, 1989, pp. 465-508.

[10] Sato, M. and Shintani, T., On zeta functions associated with prehomogeneous vector 
spaces, Ann. of Math., 100 (1974), 131-170.

[11] Shimura, G., On modular forms of half integral weight, Ann. Math., 97 (1973), 440-481.

[12] Shintani, T., On zeta-functions associated with the vector space of quadratic forms, J. Fac. Sci. Univ. Tokyo, Sec. Ia, 22 (1975), 25-66.

113] Tate, J., Fourier analysis on number fields and Hecke's zeta functions, in Cassels, J. and Fröhlich, A., Algebraic Number Theory, Academic Press, New York, 1967.

[14] Weil, A., Adeles and Algebraic Groups, Birkhäuser, Boston, 1982.

[15] Weil, A., Basic Number Theory, Springer Verlag, Berlin, Heidelberg, New York, 1974.

[16] Yukie, A., On Shintani zeta function for the space of binary quadratic forms, Math. Ann., 292 (1992), 355-374.

[17] Zagier, D., Nombres de classes et formes modulaires de poids $3 / 2$ C. R. Acad. Sc. Paris, 281 (1975), 883-886.

Department of Mathematics

Temple University

Philadelphia, PA 19122

USA 